SLAC-R-1006

\title{
LCLS Ultrafast Science Instruments II (LUSI-II) Proposal
}




\section{LCLS Ultrafast Science Instruments II (LUSI-II) Proposal}

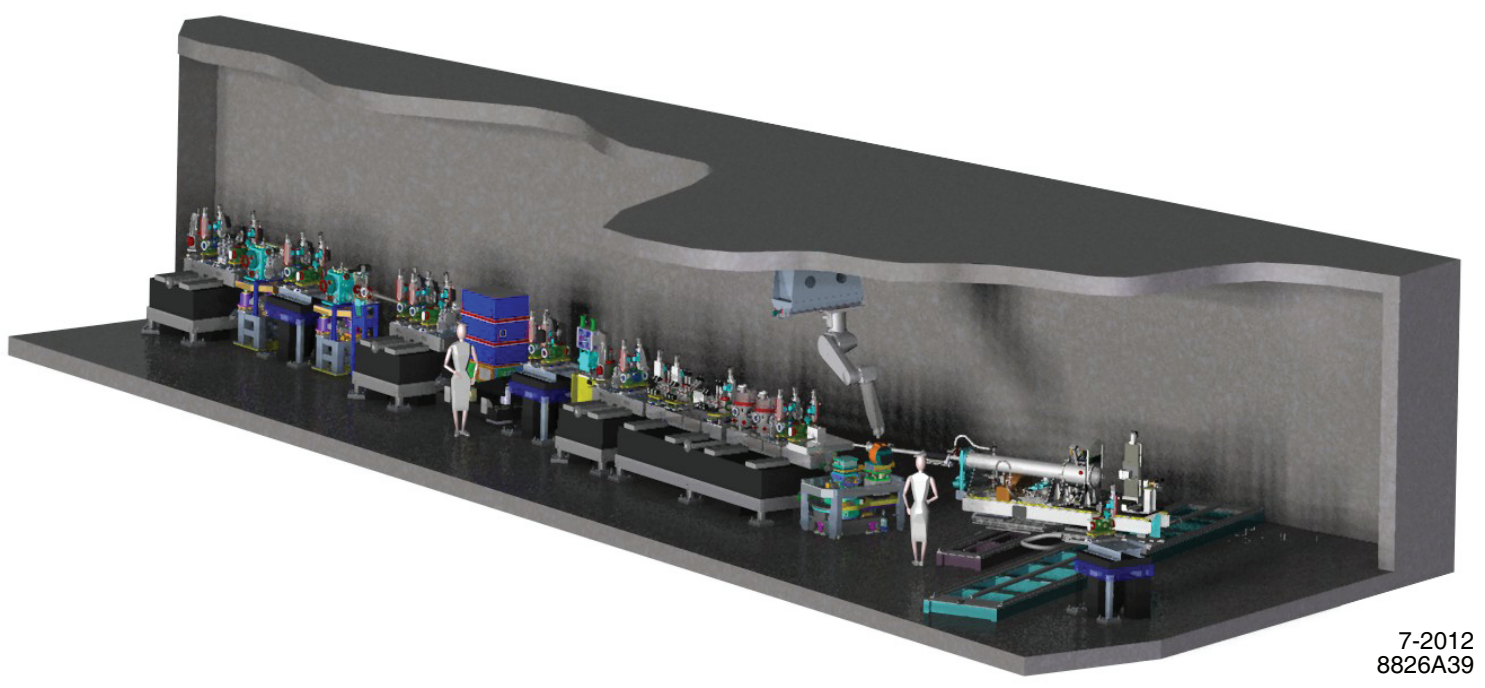

DEPARTMENT OF ENERGY

SLAC NATIONAL ACCELERATOR LABORATORY

April 2013

\section{SLAC-R-1006}

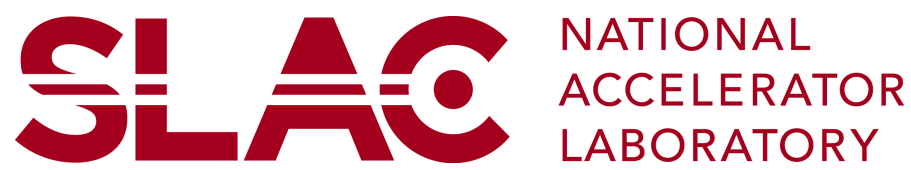




\section{Authors}

John Arthur, Bronwyn Barnett, Uwe Bergmann, Christoph Bostedt, Sebastien Boutet, John Bozek, Axel Brunger, Philip Bucksbaum, Glennda Chui, Daniele Cocco, Thomas Devereaux, Yuantao Ding, Persis Drell, Hermann Dürr, David Fritz, Kelly Gaffney, John Galayda, Michael Glavicic, Markus Guehr, Jerome Hastings, Mark Hartney, Philip Heimann, Keith Hodgson, Zhirong Huang, Chi-Chang Kao, Nicholas Kelez, Steve Kivelson, Ingolf Lindau, Anders Nilsson, David Reis, Aymeric Robert, Michael Rowen, David Rugg, William Schlotter, Marvin Seibert, Z-X Shen, Joachim Stöhr, Mariano Trigo, Joshua Turner, Soichi Wakatsuki, William White, Juhao Wu, Garth Williams, Vittal Yachandra, Junko Yano

Published by:

\section{SLAC National Accelerator Laboratory}

2575 Sand Hill Road

Menlo Park, CA 94025

This document, and the material and data contained therein, was developed under sponsorship of the United States Government. Neither the United States nor the Department of Energy, nor the Leland Stanford Junior University, nor their employees, nor their respective contractors, subcontractors, or their employees, makes any warranty, express or implied, or assumes any liability of responsibility for accuracy, completeness or usefulness of any information, apparatus, product or process disclosed, or represents that its use will not infringe privately owned rights. Mention of any product, its manufacturer, or suppliers shall not, nor is intended to imply approval, disapproval, or fitness for any particular use. A royalty-free, nonexclusive right to use and disseminate same for any purpose whatsoever, is expressly reserved to the United States and the University. 


\section{TABLE OF CONTENTS}

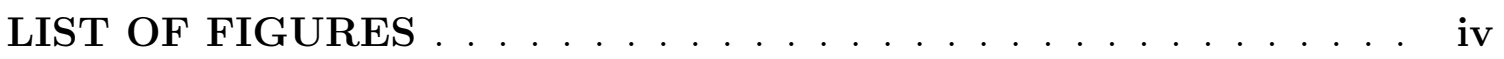

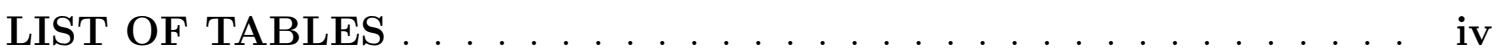

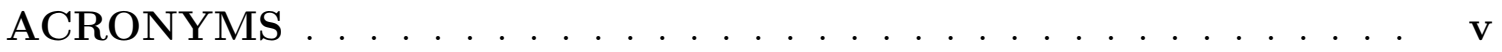

1. Executive Summary . . . . . . . . . . . . . . . . 1-1

$1.1 \quad$ Cost \& Schedule . . . . . . . . . . . . . . . . . . . . . 1-2

1.2 Acquisition Strategy . . . . . . . . . . . . . . . . . . 1-2

2. Introduction . . . . . . . . . . . . . . 2-1

2.1 The National and International Context . . . . . . . . . . . . 2-2

2.2 What Have We Learned from Three Years of LCLS Operations? 2-3

2.3 What Have We Learned from Three Years of LCLS Science? . . 2-5

2.4 Instruments at the LCLS Complex . . . . . . . . . . . . 2-7

3. Science Drivers . . . . . . . . . . . . . . . 3-1

3.1 The Role of Complexity in Materials Properties . . . . . . . . 3-1

3.1.1 Exploring the Complex Interplay Between Distinct Orders in Quantum Materials . . . . . . . . . . . . 3-1

3.1.2 Energy Flow in Systems Near and Far from Equilibrium 3-4

3.1.3 Understanding How Disorder and Dynamics Determine the Properties of Real Materials . . . . . . . . . . . . 3-6

3.2 Understanding and Controlling Photochemical Transformations on the Molecular Level . . . . . . . . . . . . . . . . . . . . 3-9

3.2.1 Determining the Design Rules Used by Proteins to Control Chemical Transformations . . . . . . . . . . 3-11

3.2.2 Converting Light to Fuel and Electricity Using Coordination Complexes . . . . . . . . . . . . . . . . 3-11

3.2.3 Elucidating the Mechanism of Photosynthetic Water Splitting . . . . . . . . . . . . . . . . . . 3-12

3.2.4 Understanding Nature's Photoprotection Mechanisms . . 3-14 
3.3 De novo Structure Determination of Challenging Biological

Targets . . . . . . . . . . . . . . . . . . . . . . . 3-17

3.3.1 HIV Vaccines . . . . . . . . . . . . . . . . . . . 3-17

3.3.2 Controlling Neurodegenerative Diseases . . . . . . . . . 3-18

3.3.3 New Capabilities Enabled by LCLS-II . . . . . . . . . 3-19

4. Instrumentation . . . . . . . . . . . . . . . . . 4-1

4.1 Angström X-ray Experiments ( $\AA$ XE) Instrument . . . . . . . . . 4-3

4.1 .1 Introduction . . . . . . . . . . . . . . . . 4-3

4.1 .2 Instrument Concept . . . . . . . . . . . . . . . . . . 4-3

4.1 .3 Specifications . . . . . . . . . . . . . . . . . . 4-4

4.1.4 Role in the LCLS Complex . . . . . . . . . . . . 4 4-4

4.2 Soft X-ray Toolbox (SXT) Instrument . . . . . . . . . . . . . . 4-6

4.2 .1 Introduction . . . . . . . . . . . . . . . 4 4-6

4.2 .2 Instrument Concept . . . . . . . . . . . . . . . 4-6

4.2 .3 Specifications . . . . . . . . . . . . . . . . . . . 4-8

4.2.4 Role in the LCLS Complex . . . . . . . . . . . . 4-8

4.3 Nanocrystal X-ray Diffraction (NXD) Instrument . . . . . . . 4-9

4.3 .1 Introduction . . . . . . . . . . . . . . . . . . . . 4 4-9

4.3.2 Instrument Concept . . . . . . . . . . . . . . . . . . 4-9

4.3 .3 Specifications . . . . . . . . . . . . . . . . . . . 4-10

4.3.4 Role in the LCLS Complex . . . . . . . . . . . . . . 4-11

4.4 Soft X-ray Ultra (SXU) Instrument . . . . . . . . . . . . . . . . 4-12

4.4 .1 Introduction . . . . . . . . . . . . . . . . 4-12

4.4 .2 Instrument Concept . . . . . . . . . . . . . . . . . . . . 4-12

4.4 .3 Specifications . . . . . . . . . . . . . . . . . . . . . . . . . 4-13

4.4.4 Role in the LCLS Complex . . . . . . . . . . . . . . 4-13

4.5 Soft X-ray Mono (SXM) Instrument . . . . . . . . . . . . . 4-14

4.5 .1 Introduction . . . . . . . . . . . . . . . . . . 4-14

4.5.2 Instrument Concept . . . . . . . . . . . . . . . . . 4-14

4.5 .3 Specifications . . . . . . . . . . . . . . . . . 4-15

4.5.4 Role in the LCLS Complex . . . . . . . . . . . . 4-15

5. Potential enhancements . . . . . . . . . . . . . . 5-1

5.1 Nanocrystal X-ray Diffraction II (NXD-II) Instrument . . . . . 5-1

5.1 .1 Introduction . . . . . . . . . . . . . . . 5-1

5.1 .2 Instrument Concept . . . . . . . . . . . . . . . . 5-1

5.1 .3 Specifications . . . . . . . . . . . . . . . . . 5-2

$5.2 \quad$ Hard X-ray IXS . . . . . . . . . . . . . . . . . . . . . . 5-2

6. Management . . . . . . . . . . . . . 6-1 
6.1 Project Management . . . . . . . . . . . . . . . . 6-1

6.2 Organization . . . . . . . . . . . . . . . . 6-2

6.3 Schedule ....................... 6- . . . . . . . . . . . .

6.4 Cost . . . . . . . . . . . . . . . . . . 6 6-3

6.5 Environment, Safety and Health . . . . . . . . . . . . . . 6-3

6.6 Risk Management . . . . . . . . . . . . . . . . . 6-3

6.6.1 Technical Risks . . . . . . . . . . . . . . . 6-4

6.6.2 Cost Risks . . . . . . . . . . . . . . . . . . . 6-4

6.6.3 Schedule Risks . . . . . . . . . . . . . . . . 6-4

REFERENCES . . . . . . . . . . . . . . . . . 7-1 


\section{LIST OF FIGURES}

\section{Figure}

2.1 Evolution of the LCLS complex. . . . . . . . . . . . . . . 2-2

2.2 Planned x-ray FEL facilities worldwide. . . . . . . . . . . . 2-3

3.1 Phase diagram for high-temperature superconductors. . . . . . . . . 3-2

3.2 Ultrafast laser heating of magnetic solids. . . . . . . . . . . . . 3-5

3.3 Femtosecond x-ray diffuse scattering. . . . . . . . . . . . . . 3-6

3.4 Ultrafast laser heating of magnetic solids. . . . . . . . . . . . . . . . 3-8

3.5 Schematic of an artificial photosynthesis system. . . . . . . . . . 3-10

3.6 The Kok $S$-state cycle for photosynthetic water oxidation. . . . . . 3-14

3.7 Suggested photoprotection mechanism of the nucleobase thymine. . 3-16

3.8 HIV cryo-EM density maps. . . . . . . . . . . . . . . 3-17

3.9 Entry of the HIV particle into the human host cell. . . . . . . . . . 3-18

3.10 X-ray structures of amyloid fibril cross-beta spines. . . . . . . . . . 3-19

4.1 LCLS-II experimental hall instrument layout. . . . . . . . . . . . . . 4-2

4.2 Conceptual layout of the AXE instrument. . . . . . . . . . . . . . . 4-4

4.3 SXT optics layout. . . . . . . . . . . . . . . . . . . . . . . . . . . . . . .

4.4 Conceptual layout of the NXD instrument. . . . . . . . . . . . 4-10

\section{LIST OF TABLES}

\section{$\underline{\text { Table }}$}

2.1 LCLS proposal and experiment allocation summary. . . . . . . . . 2-4

2.2 LCLS instruments at completion of LUSI-II. . . . . . . . . . . . . 2-7

4.1 Specifications for the AXE instrument. . . . . . . . . . . . . . . . 4-5

4.2 Specifications for the SXT instrument. . . . . . . . . . . . . 4-8

4.3 Specifications for the NXD instrument. . . . . . . . . . . . . . . . . . . . . . 41

4.4 Specifications for the SXU instrument. . . . . . . . . . . . . . . . . . . . . . . 43

4.5 Specifications for the SXM instrument. . . . . . . . . . . . 4-15

5.1 Specifications for the NXD-II instrument. . . . . . . . . . . 5-2

5.2 Specifications for the IXS spectrometers on the XCS instrument. . . 5-3

6.1 Major milestone schedule. . . . . . . . . . . . . . 6-3

6.2 Fiscal year project funding profile assuming a TPC of $\$ 100 \mathrm{M}$. . . 6-3 


\section{ACRONYMS}

AMO

ARPES

ÅXE

BESAC

BOA

$\mathrm{BZ}$

CD

CDW

CXI

CXDI

DNA

EM

ES\&H

FACET

FEL

FWHM

FY

HIV

IXS

$\mathrm{KB}$

LCLS

LUSI

MAD

MEC

MECI

NMR

NXD

OPC

RIXS

RXD

R\&D

SAD

SASE

SAXS

SLAC

SR
Atomic, Molecular \& Optical science instrument

Angle-resolved Photoemission Spectroscopy

Ångström X-ray Experiments instrument

Basic Energy Sciences Advisory Committee

Born-Oppenheimer Approximation

Brillouin Zone

Critical Decision

Charge Density Wave

Coherent X-ray Imaging instrument

Coherent X-ray Diffractive Imaging

Deoxyribonucleic Acid

Electron Microscopy

Environment, Safety and Health

Facility for Advanced Accelerator Experimental Tests

Free-Electron Laser

Full Width at Half Maximum

Fiscal Year

Human Immunodeficiency Virus

Inelastic X-ray Scattering

Kirkpatrick-Baez

Linac Coherent Light Source

LCLS Ultrafast Science Instruments project

Multi-wavelength Anomalous Dispersion

Matter in Extreme Conditions instrument

Matter in Extreme Conditions instrument project

Nuclear Magnetic Resonance

Nanocrystal X-ray Diffraction instrument

Other Project Costs

Resonant Inelastic X-ray Scattering

Resonant X-ray Diffraction

Research and Development

Single-wavelength Anomalous Dispersion

Self-Amplified Spontaneous Emission

Small Angle X-ray Scattering

SLAC National Accelerator Laboratory

Synchrotron Radiation 


$\begin{array}{ll}\text { STM } & \text { Scanning Tunneling Microscopy } \\ \text { SXM } & \text { Soft X-ray Mono instrument } \\ \text { SXR } & \text { Soft X-Ray instrument } \\ \text { SXT } & \text { Soft X-ray Toolbox instrument } \\ \text { SXU } & \text { Soft X-ray Ultra instrument } \\ \text { TEC } & \text { Total Estimated Cost } \\ \text { TDMD } & \text { Time Dependent Momentum Distribution } \\ \text { TPC } & \text { Total Project Cost } \\ \text { UHV } & \text { Ultra High Vacuum } \\ \text { UV } & \text { Ultraviolet } \\ \text { WAXS } & \text { Wide Angle X-ray Scattering } \\ \text { XAS } & \text { X-ray Absorption Spectroscopy } \\ \text { XCS } & \text { X-ray Correlation Spectroscopy instrument } \\ \text { XES } & \text { X-ray Emission Spectroscopy } \\ \text { XFEL } & \text { X-ray Free-Electron Laser } \\ \text { XPCS } & \text { X-ray Photon Correlation Spectroscopy } \\ \text { XPP } & \text { X-ray Pump-Probe instrument }\end{array}$




\section{Executive Summary}

We propose to build a suite of science instruments that will allow users to take full advantage of LCLS-II, an expansion of the existing Linac Coherent Light Source that is expected to produce first light in 2018. LCLS-II will add two undulator sources for producing x-ray laser beams, tripling the capacity of the current facility. The instrument construction project proposed here, LCLS Ultrafast Science Instruments II (LUSI-II), will greatly enhance the capabilities of LCLS and keep it at the forefront of an intensifying international race to build and operate x-ray free-electron laser (XFEL) facilities. The proposed instruments have been identified in collaboration with the scientific community $[1,2]$ and are in alignment with the DOE mission of managing world-leading basic research programs and stewarding unique user facilities. We have further considered the experience gained from the first three years of LCLS user operation and science, which have been extremely successful. The proposed instruments will complement those currently operated at LCLS and take full advantage of the enhanced capabilities to be delivered by LCLS-II. In particular, we propose to build in this project:

- A multipurpose hard x-ray instrument covering the spectral range up to $18 \mathrm{keV}$, suitable for a wide range of scattering, diffraction, and spectroscopy studies.

- A soft x-ray facility consisting of three instruments covering the $250-2,500 \mathrm{eV}$ spectral range that will provide increased capabilities and capacity for soft x-ray science.

- A dedicated instrument for performing nanocrystal x-ray diffraction, which has been identified as the first high-impact application of LCLS.

In addition to tripling the current capacity, the new instruments' enhanced capabilities and spectral range will allow new research previously not possible. We recently held a workshop with scientists from the fields of materials, chemical, and biological sciences [1], and from these fields we have identified three science thrust areas that will drive the instrumentation portfolio proposed here. They are: 
- The role of complexity in materials properties.

- Understanding and controlling photochemical transformations on the molecular level.

- De novo structure determination of challenging biological targets.

Examples from each area are discussed in this proposal. Although some can be directly linked to a specific instrument or technique, others will take advantage of more than one new capability in our proposed portfolio. Given the relatively short time since the beginning of LCLS operations, we are still on a steep learning curve. As more results come in, some capability upgrades that were not in the LCLS-II baseline design, including self seeding, polarization, and pulse control, as well as increased peak power, spectral range, and repetition rate, will be prioritized. Although these upgrades are considered in both our science discussions and our planned instrument build-out, they are not part of this proposal.

The international community has taken notice of the enormous success of LCLS. The SACLA facility is already operating in Japan, and others in Germany (European XFEL), Korea, and Switzerland will come online in the next three to five years. With the funding of LCLS-II, the United States is in a unique position to maintain its leadership in XFEL-based science, and the instrumentation in this proposal will be crucial for realizing that goal.

\subsection{Cost \& Schedule}

The LUSI-II total project cost (TPC) is in the $\$ 80 \mathrm{M}-\$ 100 \mathrm{M}$ range. A six-year construction schedule beginning in FY 2015 is proposed.

\subsection{Acquisition Strategy}

The lead contractor for acquisition of the LUSI-II project is Stanford University, which operates the SLAC National Accelerator Laboratory for the US Department of Energy. 


\section{Introduction}

We propose to build a suite of science instruments that will allow users to take full advantage of LCLS-II, an expansion of the existing LCLS that is expected to produce first light in 2018. LCLS-II will add a second high-energy electron beam that will feed two variable-gap undulator sources for producing x-ray laser beams, tripling the capacity of the current facility. LCLS-II will operate independently from LCLS-I and will be optimized for producing radiation in both the soft (250-2500 eV) and hard (5-18 keV) x-ray regimes via purpose-built undulator systems.

The instrument construction project proposed here, LUSI-II, will deliver five stateof-the-art scientific instruments (two hard x-ray and three soft x-ray) utilizing the LCLS-II undulator sources. These instruments will greatly enhance the capabilities of LCLS and keep it at the forefront of an intensifying international race to build and operate XFEL facilities. The proposed instruments have been identified in collaboration with the scientific community $[1,2]$ and are in alignment with the DOE mission of managing world-leading basic research programs and stewarding unique user facilities.

Figure 2.1 schematically illustrates the evolution of the LCLS complex from its current state to its future state at completion of the LCLS-II and LUSI-II projects. Presently, the LCLS operates two soft and four hard x-ray instruments with a single fixed-gap undulator source in a serial fashion. The LCLS-II project will construct a variable-gap undulator system that is optimized for producing soft $\mathrm{x}$-rays with a high-energy electron beam $(>10 \mathrm{GeV})$. The performance of this source will exceed that of the LCLS-I source in the soft x-ray spectrum. Due to the enhanced LCLS-II soft x-ray source properties, all future soft x-ray activities at the LCLS complex will be performed at the three soft x-ray instruments proposed here. 


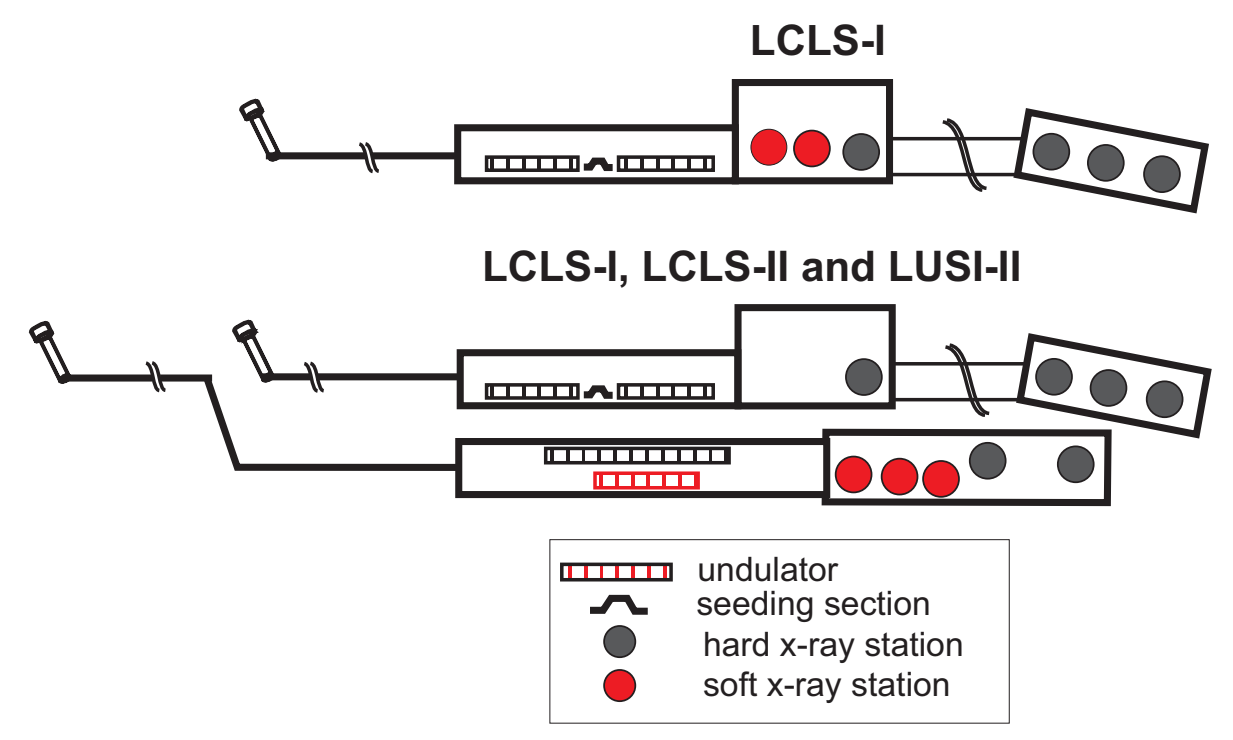

Figure 2.1: Schematic illustration of the evolution of the LCLS complex*.

\subsection{The National and International Context}

The 2009 BESAC report Next Generation Photon Sources for Grand Challenges in Science and Energy [3] specifically identified and addressed the need for new photon sources to meet those challenges. Of the suggested photon source portfolio, XFELs were identified as the most advanced tools for discovery-class science in condensed matter and materials physics, chemistry, and biology. The first three years of LCLS operation have been extremely successful and BES has funded LCLS-II as a key component of its strategy to advance its mission through building and operating world-class light sources. The international community is also moving forward aggressively. The SACLA facility started operations in Japan in 2011, and new facilities in Germany, Korea, and Switzerland are expected to come on line in the next three to five years. With the funding, construction, and operation of LCLS-II, the United States is in a unique position to maintain our leadership in XFEL-based science, and approval of this instrumentation proposal is crucial in realizing that goal. The LUSI-II project will ensure that the science community can make full use of the two new undulator sources provided by LCLS-II, which are planned to produce first light in 2018. Figure 2.2 compares undulator sources and XFEL experimental stations planned worldwide and expected to be operational by $2020 .^{\dagger}$ The figure shows that the instrumentation proposed here can ensure that the US remains internationally competitive.

\footnotetext{
*LCLS-I hard x-ray instruments delivered under LUSI-I and MECI projects. SXR instrument delivered under the SXR collaboration

${ }^{\dagger}$ Not included are XFELs that operate in the soft x-ray and lower energy regime only (fundamental $<500 \mathrm{eV})$.
} 


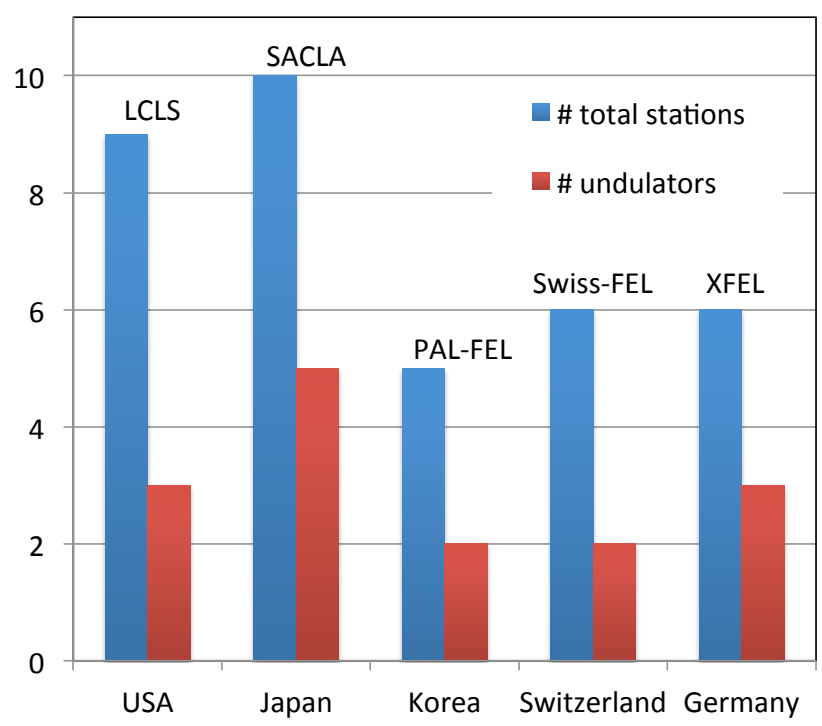

Figure 2.2: Snapshot of worldwide X-FEL facilities expected to be operational in 2020, labeled by facility names and the host country. For LCLS we have assumed the fully instrumented LCLS-II scenario.

\subsection{What Have We Learned from Three Years of LCLS Operations?}

In the past three years we have gained insight into managing the complexity of LCLS operations and ways to use beam time most efficiently - lessons that have been incorporated into this proposal for LCLS-II instrumentation. There are important differences in the way XFELs and synchrotrons operate. First, a synchrotron based on a storage ring can provide x-rays to 60 or more experimental stations at once. But an XFEL based on a linear accelerator can provide beam to only a few experiments at a time, depending on the number of undulators it operates. This puts stringent limits on the number of experiments performed and the amount of beam time per experiment. In our first runs at LCLS, we learned that it is more efficient to operate two experiments in alternating 12-hour shifts rather than one experiment for 24-hour shifts, as is common at synchrotron facilities. This requires that each XFEL undulator source have at least two experimental stations that can be operated independently, and ideally at least one additional station to allow time for setting up the next experiment and disassembling the previous one. Therefore we plan to eventually operate up to six instruments at LCLS-II - three for each of the two undulator sources.

We have also learned that XFEL experiments in general are significantly more complex, as they often involve the integration of pump laser sources, more elaborate x-ray optics and sample environments, and more complex diagnostic tools such as 
detectors, spectrometers, etc. This is reflected in the planned layout of the LCLS-II experimental hall and shown in the more detailed instrument descriptions in Chapters 4 and 5 .

The instruments proposed here will draw on the experience gained during the first three years of operation to greatly expand on the science performed at LCLS in both the hard x-ray and soft x-ray regimes. The first instrument operational at LCLS was the atomic, molecular and optical physics instrument (AMO), which started userassisted commissioning in late 2009. An additional five instruments have since been phased in: Soft X-ray Materials Science (SXR), X-ray Pump Probe (XPP), Coherent X-ray Imaging (CXI), Matter in Extreme Conditions (MEC), X-ray Correlation Spectroscopy (XCS). AMO and SXR operate in the soft x-ray regime below $2 \mathrm{keV}$; the other four operate in the hard $\mathrm{x}$-ray regime up to $10 \mathrm{keV}$.

Table 2.1 shows the number of scheduled and proposed experiments for each of the current LCLS instruments. It shows that the two most heavily subscribed and operated instruments are XPP and CXI. As discussed in Chapters 4 and 5, the new hard x-ray instruments proposed here will expand on the science currently performed on these instruments. We further propose to build three soft x-ray instruments that will serve and expand the current AMO and SXR user communities.

Table 2.1: LCLS proposal and experiment allocation summary.

\begin{tabular}{|r|c|c|c|c|c|c|c|c|}
\hline \hline & Run 1 & Run 2 & Run 3 & Run 4 & Run 5 & Run 6 & Run 7 & Run 8 \\
\hline Proposals & & & & & & & & \\
Received & Sept'08 & May'09 & Nov'09 & Jun'10 & Jan'11 & Sept'11 & Jul'12 & Jan'13 \\
\hline AMO & 28 & 24 & 16 & 25 & 15 & 15 & 17 & 21 \\
SXR & & 38 & 32 & 31 & 18 & 28 & 23 & 19 \\
XPP & & & 59 & 35 & 34 & 27 & 35 & 47 \\
CXI & & & & 25 & 29 & 36 & 45 & 57 \\
MEC & & & & & 12 & 18 & 19 & 22 \\
XCS & & & & & 6 & 10 & 13 & 9 \\
\hline Run Totals & 28 & 62 & 107 & 116 & 114 & 135 & 152 & 175 \\
\hline Experiments & & & & & & & & \\
Performed & Oct'09- & May'10- & Oct'10- & Jun'11- & Nov'11- & Jun'12- & Jan'13- & Jul'13 \\
\hline AMO & 11 & Sep'10 & Mar'11 & Oct'11 & May'12 & Dec'12 & Jul'13 & - Mar'14 \\
\hline SXR & & 13 & 5 & 7 & 4 & 7 & 5 & 3 \\
XPP & & & 5 & 6 & 4 & 5 & 7 & 6 \\
CXI & & & 4 & 6 & 8 & 7 & 12 & 8 \\
MEC & & & & & 2 & 4 & 4 & 5 \\
XCS & & & & & 2 & 2 & 3 & 2 \\
\hline Run Totals & 11 & 23 & 25 & 25 & 29 & 33 & 42 & 35 \\
\hline \% Scheduled & $39 \%$ & $37 \%$ & $23 \%$ & $22 \%$ & $25 \%$ & $25 \%$ & $28 \%$ & $20 \%$ \\
\hline \hline
\end{tabular}




\subsection{What Have We Learned from Three Years of LCLS Science?}

Science magazine selected the successful lasing of the LCLS as one of the top 10 breakthroughs of 2009, and three years later an LCLS user experiment (see below) made this prestigious list for the first time. In fact, the user community has enthusiastically responded to the revolutionary new capabilities provided by LCLS's ultrashort and ultrabright x-ray pulses, as evidenced by the scientific productivity and strong and growing user demand. Starting with the very first experiments, there has also been an extremely fruitful interaction between the users and the LCLS/accelerator staff to push the LCLS capabilities to new frontiers beyond the baseline design. In the first three years of LCLS operations, numerous scientific breakthroughs have been achieved. ${ }^{\ddagger}$

The first experiments focused on AMO studies, in particular how the ultra-intense femtosecond x-ray pulses interact with atoms and molecules. Scientists were able to create exotic states including hollow atoms, supercharged atoms, double core hole excitations, x-ray-induced solid-density plasmas and nanoplasmas and an atomic xray laser [4-16]. While these results are interesting in their own right, they are also critical to finding new ways to use an XFEL to investigate scientifically or technologically important samples. One of our proposed instruments, Soft X-ray Ultra (SXU, see Section 4.4), will focus on this line of research with capabilities based on what we have learned in these experiments.

One of the primary motivations for the development of XFELs was the promise of coherent diffraction imaging of single molecules. Although significant progress has been made, this goal remains challenging. Diffractive imaging of the mimivirus [17], and more recently of aerosols [18], represents a critical first step towards the ultimate goal of imaging non-periodic objects with near-atomic resolution. However, significant developments are still required. The increased pulse energy and control of LCLS-II in the 2-2.5 keV photon energy range at the SXU instrument will prove crucial here. If successful, this capability will transform our understanding of nonperiodic objects of relevance to catalysis, energy storage, structural biology, and health as well as many other fields.

Examples of recent studies using SXR, the soft x-ray monochromatic instrument at LCLS, include the real-time observation of bond breaking between a carbon monoxide molecule and the surface of a ruthenium catalyst [19], the imaging of ferromagnetic nanoscale domains [20], the dynamics of charge ordered nickelates [21] and optically induced ultrafast spin reversal in a ferrimagnet [22]. Our two proposed soft x-ray instruments, Soft X-ray Toolbox (SXT) and Soft X-ray Mono (SXM), will build on

\footnotetext{
${ }^{\ddagger}$ For a list of LCLS publications see

https://portal.slac.stanford.edu/sites/lcls_public/Pages/Publications.aspx
} 
the lessons learned from these and related experiments and dramatically improve our current capabilities and capacity for such studies (see Sections 4.2 and 4.5). In particular, we will focus on spectroscopic studies including various forms of photon-in photon-out techniques (see Chapter 4). Typically these techniques will be performed in a pump-probe mode, where the sample will be first manipulated with an excitation pulse (e.g. optical, THz, magnetic field or x-ray) and then characterized with an ultrashort soft x-ray probe pulse. In Sections 3.1 and 3.2, we will discuss science opportunities that drive the SXT and SXM instrumentation. Besides techniques also known at synchrotrons, such as x-ray absorption (XAS), emission spectroscopy (XES), and momentum-resolved resonant inelastic x-ray scattering (RIXS), we propose to develop new schemes for stimulated scattering experiments (see Section 4.2).

One of the most heavily scheduled instruments at LCLS is XPP, the multipurpose hard x-ray pump probe instrument (see Table 2.1). It is used broadly for materials science, chemistry, and biology as well as for fundamental quantum optics experiments, as has been shown in a recent study on x-ray and optical wave mixing [23]. Hard x-rays are used for coherent scattering as well as spectroscopy of bulk samples, and studies at XPP include the ultrafast photovoltaic response in ferroelectric nanolayers [24] and atomic-scale order in liquids and glasses by high-contrast x-ray speckle [25]. Earlier, the spatial and temporal coherence properties of single-shot hard x-ray pulses had been studied, as these are critical properties for a variety of scattering experiments [26]. Overcoming the temporal jitter between pump and probe has been a limitation at XPP and other instruments, but new schemes to overcome this have recently been developed [27,28], including a time-sorting system with few-femtosecond precision in the hard x-ray regime [29]. The Angström X-ray Experiments ( $\AA \mathrm{XE})$ instrument proposed here is our highest priority and is based on the same multipurpose philosophy as XPP with further enhanced capabilities (see Section 4.1).

This year Science magazine voted the LCLS-based serial crystallography work on a protein called cathepsin B [30] onto its distinguished top-10 scientific breakthrough list for 2012. Cathepsin B is an inactive "precursor" form of an enzyme that is key for the survival of the single-celled parasite that causes African sleeping sickness, responsible for more than 10,000 deaths every year. The work used the "diffraction before destruction" approach pioneered at LCLS [31] to determine the precursor's structure, which includes a kind of molecular safety cap that deactivates it. That information could help scientists find a drug to tie up the active form of the enzyme. In serial crystallography, the ultrashort x-ray pulses from LCLS create thousands of single-shot diffraction patterns from a stream of very small crystals (from $100 \mathrm{~nm}$ to a few microns in size). With sufficient redundancy, these diffraction patterns from randomly oriented crystals can be used to determine a three-dimensional structure. This method has been shown to yield the intact structure of lysozyme at 1.9 Angström resolution [32]. Using a combination of diffraction and x-ray emission spectroscopy, it was also shown very recently that this femtosecond-pulse serial crys- 
tallography approach can provide the intact chemical structure of the manganese cluster in Photosystem II [33] at room temperature. This complex is responsible for splitting water in photosynthesis and has been notoriously difficult to characterize due to its high sensitivity to x-ray damage. A flurry of publications on LCLS-based serial crystallography in the last two years [30-43] has led to considerable excitement in the structural biology community and the hope that XFELs will provide transformational new methods of solving structures from challenging systems. Yet, we still have to show that a completely unknown structure can be solved de novo with this approach. Our proposed Nanocrystal X-ray Diffraction (NXD) instrument at LCLS-II will play a critical role in such work (see Sections 3.3 and 4.3).

Finally, the rapid development of accelerator and $\mathrm{x}$-ray $\mathrm{R} \& \mathrm{D}$ in the first three years of LCLS operations has led to new capabilities, including self-seeding, beam splitting, polarization control, and two-color pulses. Furthermore, we are investigating new opportunities to provide an increased pulse energy and repetition rate. These capabilities will be implemented in the coming years. The proposed instruments will be designed to take full advantage of these enhancements.

\subsection{Instruments at the LCLS Complex}

The proposed instruments will complement those currently operated at LCLS and take full advantage of the enhanced capabilities to be delivered by LCLS-II. Table 2.2 details the instrumentation set that will be realized at the completion of the LUSI-II project.

Table 2.2: LCLS instruments at completion of LUSI-II.

\begin{tabular}{|c|c|l|}
\hline \hline Undulator Source & Instrument & Specialty \\
\hline LCLS-I hard x-ray & XPP & Scattering and spectroscopy \\
LCLS-I hard x-ray & XCS & Correlation spectroscopy (monochromatic) \\
LCLS-I hard x-ray & CXI & High-intensity (imaging, nonlinear physics) \\
LCLS-I hard x-ray & MEC & High energy density, shock physics \\
LCLS-II soft x-ray & SXT & Advanced spectroscopy (XES, RIXS, stim. RIXS) \\
LCLS-II soft x-ray & SXU & High-intensity (nonlinear physics, imaging) \\
LCLS-II soft x-ray & SXM & Resonant diffraction \\
LCLS-II hard x-ray & AXE & Scattering and spectroscopy \\
LCLS-II hard x-ray & NXD & Crystallography \\
\hline \hline
\end{tabular}




\section{Science Drivers}

In the following sections we will discuss several scientific opportunities that will directly benefit from the enhanced capabilities provided by LCLS-II and the instrumentation proposed here in the LUSI-II project. More examples can be found in the LUSI-II workshop report and white paper $[1,2]$.

\subsection{The Role of Complexity in Materials Properties}

\subsubsection{Exploring the Complex Interplay Between Distinct Orders in Quantum Materials}

The complexity of the emergent phenomena in highly correlated materials may well be their defining feature. The phase diagrams of such materials are astonishingly rich, with multiple phases occurring on comparable(relatively high) energy scales (see Figure 3.1) [44]. Moreover, local forms of "fluctuating order" (i.e. significant and measurable electronic structure that resembles that in a proximate broken symmetry phase) extend well outside the corresponding phase boundaries [45]. It is increasingly clear that these orders cannot be thought of as isolated phenomena, or even strictly as "competing orders," but that rather they are "intertwined" in a complex manner, all reflecting different aspects of the underlying quantum physics of the strongly correlated electron fluid. Even precisely identifying the nature of the broken symmetry states that occur in these materials has proven to be a remarkably difficult undertaking, and disentangling the nature of the mesoscale order that arises at intermediate time and length scales, or even defining it precisely, is a still more daunting task.

This aspect of the problem has come increasingly into sharp focus as a result of recent results concerning the most studied of all highly correlated systems, the cuprate hightemperature superconductors [45]. In addition to the well-known antiferromagnetic and $d$-wave superconducting phases, it is now clear that, especially associated with the infamous pseudogap regime of the phase diagram [46], there are a variety of other broken symmetry phases including at least incommensurate charge and spin density waves and electron nematic phases. In a dramatic recent development, resonant inelastic soft x-ray (RIXS) experiments [47] and hard x-ray [48] scattering studies of 

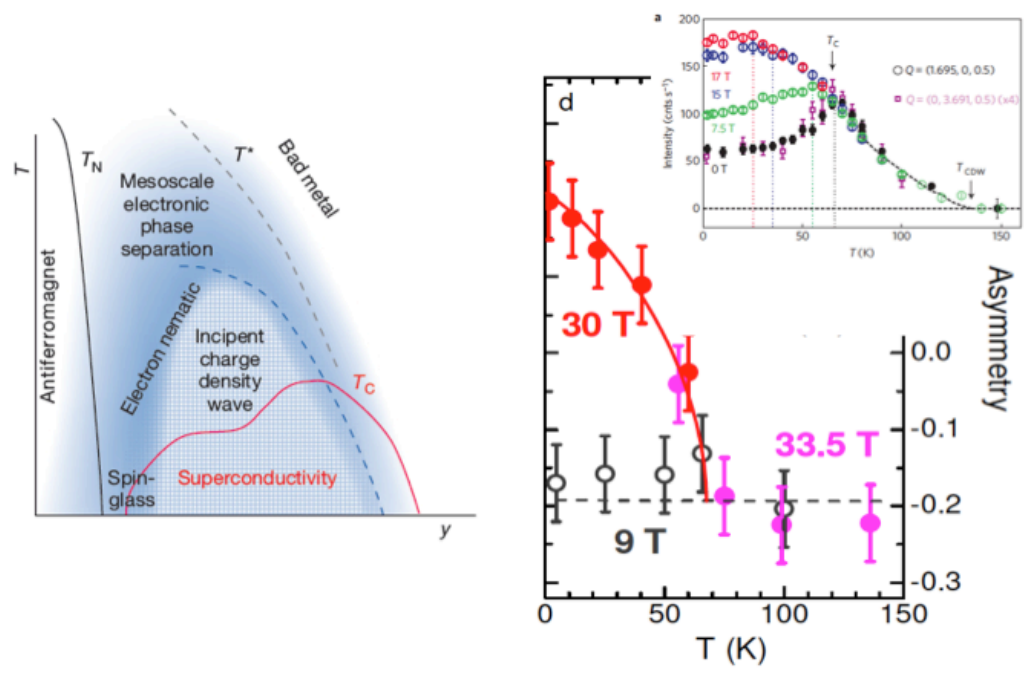

Figure 3.1: (left) Sketch of a phase diagram for high-temperature superconductors [50]. (top right) Intensity of charge ordering peak [48] (bottom right) and strength of NMR asymmetry signal [51] in magnetic fields in YBCO.

yttrium barium copper oxide (YBCO) have shown that short-range charge-density wave (CDW) order extends over a much larger range of temperatures and material parameters than was previously appreciated. That the interplay between these various orders is not a peculiarity of a particular subset of cuprate superconductors (as many previously thought) is made clear both by the increasingly broad range of cuprates in which it is seen, and by the clear analogies with the phase diagrams of the Fe-based superconductors [49], the other family of unconventional high-temperature superconductors where unidirectional spin-density wave and electron nematic orders are again strongly coupled to the superconductivity.

Unraveling the various ordering tendencies where they compete, especially where they occur as fluctuational effects with finite (even if substantial) correlation lengths and persistence times, is intrinsically hard and requires new experimental capabilities. The above-mentioned recent discovery of fluctuating CDW order in YBCO is a case in point. This material has been studied in great detail by a large number of methods for 25 years, but these strong correlations had previously escaped detection despite the fact that they give rise to a significant peak in the structure factor, are sharply defined (the full width at half maximum is less than $10 \%$ of the ordering vector), and were expected on the basis of various theoretical considerations.In contrast with most other probes, the information obtained is, ideally, both energy and momentum resolved.

Rapid development of RIXS has allowed the investigation of low-energy excitations at an energy range of $\sim 100 \mathrm{meV}$ that bear the hallmark of the symmetry-broken 
states. In addition, the combination of IXS under high pressure has provided another knob to tune emergence in materials. These equilibrium state experiments are not only important for a fundamental understanding of the novel state, but also provide complementary information for the proposed and future time domain experiments. Recently, momentum-resolved RIXS has reached sufficient energy resolution to measure single magnon spin excitations and has drawn significant attention in the field [52-54]. However, how the RIXS spectrum is related to the spin-spin correlation function remains an open question. In addition to the spin excitations, we would like to search for novel collective charge excitations associated with the striped state. As it is strongly intertwined with superconductivity, its dynamics and excitation are crucial for gaining further insights into the high $\mathrm{T}_{c}$ [45]. Recently, RIXS measurements at the $\mathrm{Cu} \mathrm{K}$-edge have revealed signatures that might be related to stripe-induced charge excitations [55]. This excitation, surprisingly, spans up to $1 \mathrm{eV}$ in energy loss.

The need for higher energy resolution cannot be overstated. The new LCLS-II instrumentation will combine this with the highest possible temporal resolution within the time-energy uncertainty. The proximity of various phases in the phase diagrams, especially near a quantum critical point, can partition the material into electronically distinct domains that experience temporal and spatial fluctuations over many time and length scales. Quasistatic and dynamic inhomogeneities are ubiquitous in correlated systems. Probes that can measure this response would reveal how, for example, the spatial fluctuations of the gap in a high- $\mathrm{T}_{c}$ superconductor are connected to the collective dynamics, or how the lattice relaxes locally in response to charge transfer that underlies Jahn-Teller phenomena in manganites and multiferroics. This would yield tremendous insights that would be felt across many areas of fundamental materials physics.

To be specific, we consider ongoing and imagined future studies of the pseudogap phase of the cuprates as illustrative examples. Some of the most exciting recent developments in the field have involved explorations of the "normal" state reached by suppressing superconductivity with high magnetic fields. Pulsed field measurements in underdoped YBCO up to $101 \mathrm{~T}$ at very low temperatures have revealed quantum oscillations characteristic of a Fermi liquid [56], but with a Fermi surface structure that is entirely different from that predicted by band theory. Likewise, the specific heat at low temperatures at fields up to $45 \mathrm{~T}$, well in excess of $\mathrm{H}_{c 2}$, is still only around $20 \%$ of its expected normal state value [57]. Recent NMR studies in fields up to $35 \mathrm{~T}$ have found evidence of a transition at around $20 \mathrm{~T}$ from a uniform state to a unidirectional ("stripe ordered") CDW state at higher fields [51]. However, the x-ray scattering studies referred to above have found evidence for CDW order even in zero field, whose amplitude (at temperatures below the superconducting $\mathrm{T}_{c}$ ) is strongly enhanced by the application of fields up to $17 \mathrm{~T}$ (see Figure 3.1). Clearly, it is essential to extend the x-ray studies to pulsed magnetic fields, at the very least so that the apparent discrepancies between different results can be resolved, and universal features across different families of cuprates can be identified. At a more profound 
level, these results clearly indicate how important it is to be able to explore in detail the nature of the phase competition that occurs at low temperatures, where distinctions between quantum phases can be drawn precisely. The requisite high fields are extremely difficult to generate statically, but can be much more readily generated as pulsed fields, necessitating time-resolved x-ray scattering studies extending to high momentum transfer that can be enabled only by the photon energy reach, $18 \mathrm{keV}$, provided by LCLS-II with high photon fluxes.

In a similar vein, a set of recent experiments has revealed preliminary evidence that, by gently tweaking one or another particular phonon mode of a cuprate, the competition between CDW and superconducting order can be greatly affected, leading to a spectacular photon-assisted enhancement of the bulk superconducting $\mathrm{T}_{c}$ in LBCO [58]. These results are all preliminary, but they give some indication of the potential to use RIXS to explore the behavior of interesting materials under conditions that can be established only for short time intervals, as shown schematically in Figure 3.2. Manifestly, LCLS-II offers the high-precision short-time x-ray scattering capabilities that will be essential to any such study.

Recently LCLS has offered the possibility of studying femtosecond time-resolved resonant x-ray scattering (RXS) and dynamical spin/charge/orbital ordering phenomena in photo-excited non-equilibrium states by directly monitoring the elastic scattering to study the associated order parameters in the time domain. Time-resolved RXS measurements at LCLS on various types of spin/charge/orbital orders in complex transition metal oxides have advanced our understanding of non-equilibrium properties of complex materials. It was discovered that the evolution of photo-excited charge orders $(\mathrm{CO})$ and spin orders $(\mathrm{SO})$ can exhibit very different behavior than their thermal evolution, providing a new pathway to control novel nanoscale electronic orders [59-61]. These results provide necessary foundations to further address deeper questions, particularly the underlying formation mechanism and the possibility of controlling the spin/charge/orbital ordering by mode-selective photon pumping using mid-IR/THz pulses. With LUSI-II instruments, time-resolved RIXS measurements near the characteristic wave vectors associated with different electronic orders can be used to track their formation in the momentum-energy-time domain. This is a new and unique type of experiment that combines RIXS and RXS in the time domain, and is well suited to LCLS.

\subsubsection{Energy Flow in Systems Near and Far from Equilibrium}

The macroscopic characteristics of a solid, such as its thermal, optical or transport properties, are determined by the available microscopic states above its lowest energy level. These slightly higher quantum states are described by elementary excitations (phonons, magnons, quasi-particles) and dictate the response of the system under external stimuli. The spectrum of these excitations, obtained typically from inelastic neutron [63] and x-ray [64-66] scattering, is the spatial and temporal Fourier 


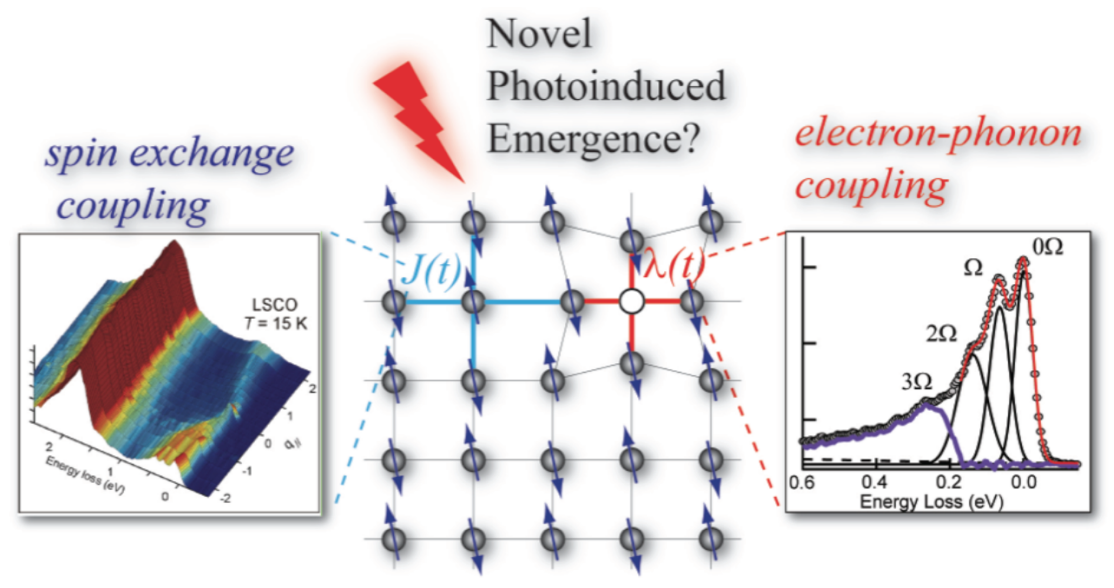

Figure 3.2: Schematic for time-domain RIXS to probe elementary excitations. (left) Soft x-ray RIXS showing magnetic excitations in underdoped cuprate [52]. (right) Observation of phonon satellites from the elastic line in 1-D cuprate [62].

transform of the density-density correlation function (fluctuations) of the system, which dictates how a perturbation propagates in space and time [67]. Access to time-resolved information on these correlations would allow us to "watch" the flow from an initial excitation into other coupled degrees of freedom, and thus could yield a more direct way to investigate the excitations of solids and their couplings both near and far from equilibrium.

By detecting the diffuse scattering of femtosecond x-ray pulses produced by an FEL, one can access large regions of the excited-state phonon dispersion relations by directly measuring these density-density correlations in the time domain. Real, timedependent correlations are created when a femtosecond laser pulse slightly quenches the phonon frequencies, producing pairs of phonons at momenta $+\mathrm{q}$ and $-\mathrm{q}$. These pairs of phonons manifest themselves as time-dependent coherences in the displacement correlations [68] that are probed directly by x-ray scattering. One can obtain spectroscopic information over large areas of reciprocal space with $<0.2 \mathrm{meV}$ energy resolution, without resolving the energy of the outgoing photon, by a Fourier transform of the femtosecond dynamics. Since the time-dependent correlations in solids are preferentially created in regions of strong electron-phonon coupling, the time-resolved approach is natural as a spectroscopic tool of low-energy collective excitations in solids, and their microscopic interactions, both in linear response and beyond. A recent experiment highlighting this potential investigated the time dependent diffuse scattering in Ge following excitation with a femtosecond optical laser (see Figure 3.3) [69]. 

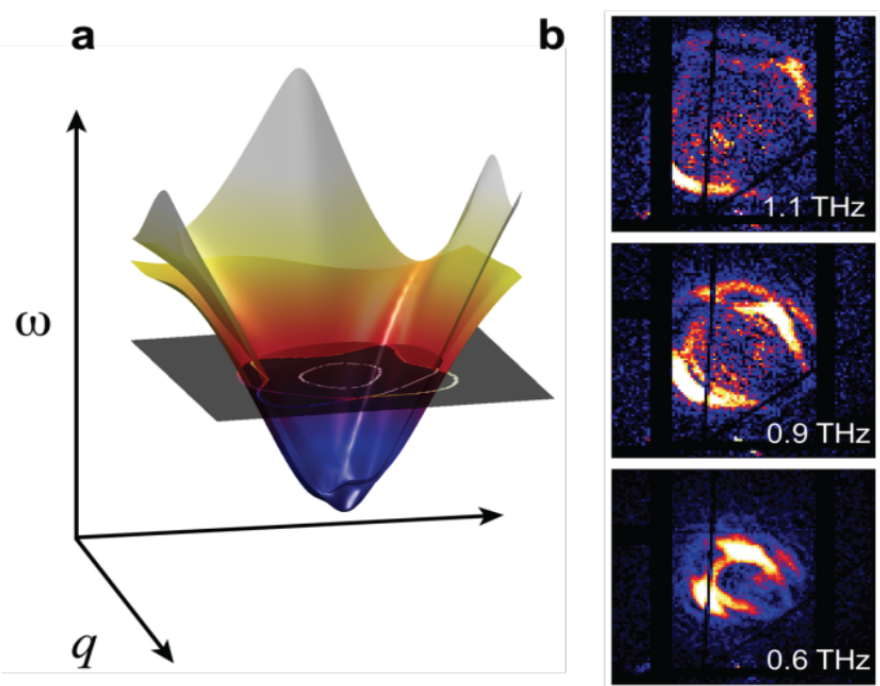

Figure 3.3: Summary of the recent XPP LCLS results for femtosecond x-ray diffuse scattering measuring $\mathrm{S}(\mathrm{q}, \mathrm{t})$ : (a) Schematic representation of the transverse acoustic phonon dispersion. (b) Intensity distribution of acoustic phonons in Ge at representative frequencies after Fourier transforming [69].

\subsubsection{Understanding How Disorder and Dynamics Determine the Prop- erties of Real Materials}

Over the last half-century or more, x-rays and neutrons have successfully unraveled the structure and dynamics of well-ordered crystalline materials by means of elastic and inelastic scattering studies. The determination of bulk crystalline structure is now routine, and based on concepts like the Brillouin zone and the associated electron, phonon, and magnon energy dispersions, a robust understanding of the electronic and magnetic structure and their dynamics has been established. It is important to note that in most cases even excited states can be described, as long as they are either a low-energy equilibrium state or a small perturbation of an equilibrium state.

One challenge is to go beyond periodic systems and equilibrium structure, and this is where XFELs shine. In this first example, we shall consider the importance of hard x-rays for the study of non-periodic systems and their dynamics. In contrast to crystalline materials, it remains a challenge to properly understand and describe non-periodic or disordered systems such as liquids, glasses, amorphous systems, and mesoporous assemblies. The description of the complexity and unique properties of disordered systems requires the determination of the degree of disorder and associated correlation lengths. Typically, conventional diffraction can be used for correlation lengths longer than $3 \mathrm{~nm}$, as is the case for polycrystals, but the determination of local short-range order below $3 \mathrm{~nm}$ has been notoriously difficult and elusive owing to the weakness of coherent diffraction signals. 
Glasses and amorphous systems exhibit an extreme form of disorder. They do not present any translational symmetry but can accommodate different local symmetries within the same system. Some of these, like the icosahedral local order, are forbidden motifs in periodic structures. The dynamics and lifetimes of these mysterious localized configurations within disordered systems have fascinated scientists for decades. They are predicted to be at the origin of some of the fundamental metastable states of matter, such as the undercooled and glassy liquid states which, together with amorphous solids, are of great interest because such materials may display novel smart functions of use in advanced technologies.

The topics and systems of interest are extremely diverse: (i) nucleation and growth of zeolites for catalysis; (ii) order-disorder phase transitions in polymers, biological systems, nanocomposites and hybrid materials; (iii) atomic diffusion in solids; (iv) complex multiscale structure of mesoporous materials; and (v) compositional order/disorder in ferroelectrics and chalcogenic glasses of interest for data storage and memory applications.

Furthermore, the intrinsic flexibility of the hard x-rays produced by LCLS-II will enable the investigation of materials of importance to many real-world applications. For example, within the aerospace and energy materials communities one will be able to study for the first time: 1) chemical-diffusion-free martensitic phase transformations in high-strength steels and titanium alloys; 2) twin formation, growth and dislocation formation at high strain rates; 3) phase transformations under extreme conditions for materials used in ballistic applications; and 4) the effects of radiation cascade damage in materials used in nuclear applications. The temporal resolution readily achievable with XFELs will allow a detailed mechanistic understanding of the above, and therefore allow science-driven optimization of these industrially important classes of materials.

Because of the possibility of increased momentum transfer, hard x-rays are particularly valuable for the study of the short-range structure of such materials. These studies furthermore benefit from the near transform-limited pulses of self-seeded FELs, which assure that the x-rays remain in phase over the entire volume of a (thick) bulk sample. LCLS-II provides the critical high photon energies (up to $18 \mathrm{keV}$ ) that provide the optimum diffraction capabilities at atomic resolution while allowing snapshots of dynamics on a timescale limited only by the pulse length.

Determining the timescales for electronic and magnetic switching is clearly important for technology. However, it also serves fundamental science: It enables us to disentangle competing interactions in the time domain, as illustrated in Figure 3.4. Exploring the materials' responses - for example, insulator-metal transitions in response to external electro-magnetic stimuli - will be instrumental in developing future oxide electronics applications [70]. Adding the spin degree of freedom brings 
new complexity (see Figure 3.4) but at the same time promises new functionality. Interestingly, for magnetism the intimate link between basic science and spintronics applications has already been demonstrated following the discovery of the giant magnetoresistance effect [71,72].

In addition, in conditions very far from equilibrium, novel electronic and magnetic phases often emerge that promote novel materials properties and functionalities. Polarized femtosecond x-ray pulses from LCLS have provided first glimpses of these new phases. A recent example is the emergence of ferromagnetic order in $\sim 10 \mathrm{~nm}$ regions of an antiferromagnetic material following laser excitation [22]. This novel spin state emerges in the far-from-equilibrium conditions during the first picosecond following laser excitation and persists for picoseconds into the subsequent near-equilibrium state. It is thought to be the enabler of so-called all-optical magnetization reversal, i.e. the potential of switching a magnetic bit in future data storage devices without the need to apply any magnetic field [73]. This concept of harvesting and ultimately controlling the emergence of new functionality far from equilibrium extends far beyond magnetic materials research. It will play a crucial role in taking advantage of quantum materials properties, such as metal-to-insulator transitions and even superconductivity, to enhance future devices. Transient states displaying materials properties not found in thermal equilibrium may be used for novel forms of quantum computing performed during the lifetime of such states. The use of hard x-rays at the highest energies $(18 \mathrm{keV})$ will enable detailed chemical structure determination crucial to the understanding of the evolution of the spin structure of these materials following laser excitation. The use of seeded x-ray pulses with full polarization control is essential in separating charge from spin responses down to the ultimate few-nm length scale [20].

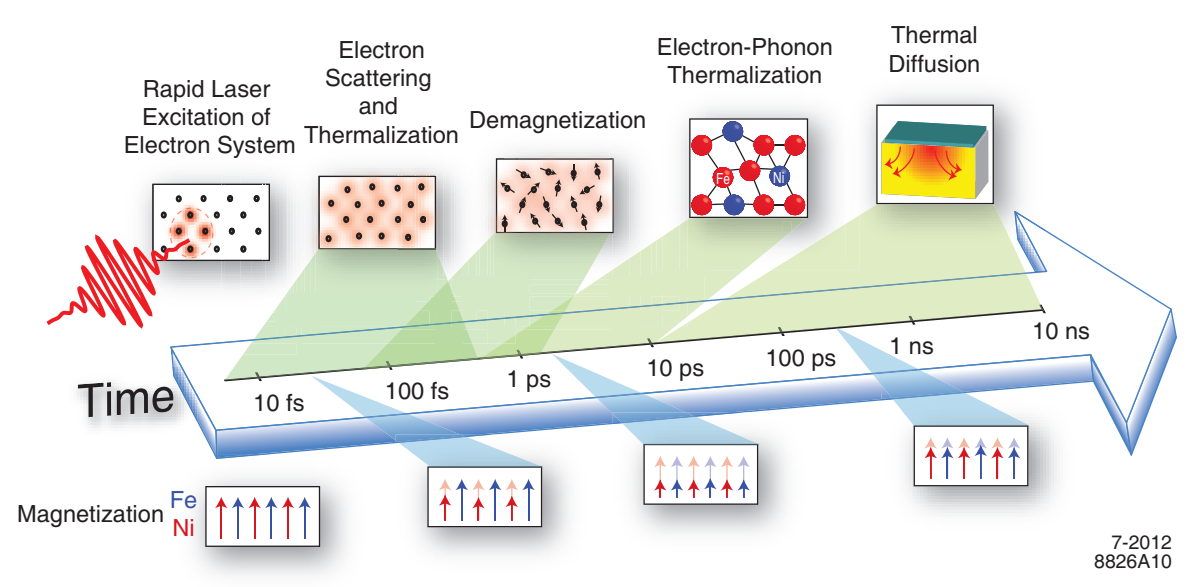

Figure 3.4: Complexity of phenomena following ultrafast laser heating of magnetic solids. The flow of energy from electrons to spins and ultimately to the lattice is accompanied by angular momentum exchange between different magnetic subsystems promising novel applications in information technology (from [74]). 


\subsection{Understanding and Controlling Photochemical Transformations on the Molecular Level}

Understanding and controlling chemical transformations on the molecular level represents one of the grand scientific challenges, and is central to generating cost-effective and environmentally benign energy sources. Such processes often occur at local catalytic reaction centers, and their tracking is aided by the atomic and chemical specificity of x-rays. Controlling chemical reactivity promises to allow the synthesis of many new, useful products, efficient and compact energy storage and release by chemical bond manipulation, and the ability to influence key biological reactions and functions.

Reactions triggered by optical photons from the sun power nearly all biological functions, either directly or indirectly. Light-driven processes also present a risk to life; the absorption of UV light by DNA can lead to mutation and cancer. In all of these cases, light absorption perturbs the valence electrons, which provide the binding forces between the nuclei and thus stabilize the molecule in a particular nuclear geometry. In the ground state, all forces among nuclei and electrons are balanced. If a photon is absorbed, the electrons reorganize, and the equilibrium is disturbed. What follows is a highly intertwined motion of nuclei and electrons. To perform a certain function, the motion of the molecule has to be directed into a particular channel. For example, a bond may need to be altered, as in the photochemical bond rotation that occurs in vision.

Light is not selective about the way it changes the shape of a molecule. So a molecule participating in a light-driven reaction must be constructed in such a way that light excitation drives it into a particular desirable channel or away from destructive channels, as happens in DNA photoprotection. Given the fast rate of intramolecular vibrational redistribution of energy, controlling photosynthetic or photovoltaic energy conversion requires controlling molecular motion on the femtosecond to manypicoseconds timescale. Figure 3.5 shows a schematic view of an artificial photosynthetic fuel cell, in which efficient conversion of light to electrochemical potential drives fuel formation. The success of such a device requires a balance between efficient energy conversion and cost-effective materials and engineering.

Despite extensive investigation, we still lack a quantitative understanding of photochemical reactivity, much less a predictive understanding. The powerful tools we use to understand chemical reactivity in the electronic ground state of molecules, such as transition state theory and density functional theory, cannot be simply extended to photochemical reactions where non-equilibrium trajectories determine the rate and reaction pathway. Controlling artificial photosynthetic and photovoltaic processes requires understanding the coupled quantum evolution of the electronics and nuclei in highly non-equilibrium configurations. 


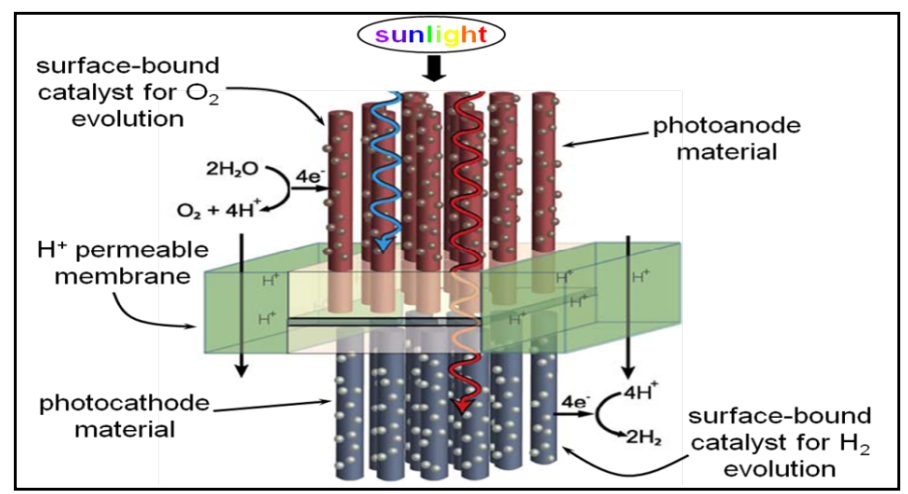

Figure 3.5: Schematic of an artificial photosynthesis system with two photoabsorbers, one at the anode and the other at the cathode, separated by a proton conductive membrane. The photoabsorbers are coated with heterogeneous catalysts providing low overpotentials for the oxidation and reduction of water.

Experimental characterization of these optically induced trajectories will benefit greatly from tools able to resolve structure, both electronic and nuclear, with a sub- $\AA$ spatial resolution. Sub- $\AA$ resolution nuclear structure requires accurate measurement of the molecular structure factor to $\mathrm{Q} \geq 10 \AA^{-1}$ and sub- $\AA$ x-ray wavelengths. The value of this high-Q structural information, and the $16 \mathrm{keV}$ x-ray photons required to efficiently acquire the information, has been clearly demonstrated in pioneering time-resolved diffuse scattering studies performed at the European Synchrotron Radiation Facility [75]. The spatial resolution of x-ray spectroscopy relies on the spatial extent of the core electronic state being excited, and benefits significantly from the expansion of the photon energy range by expanding the number of atomic edges accessible to the LCLS.

Femtosecond resolution hard and soft x-ray spectroscopy provide a powerful means of characterizing the valence electron evolution induced by optical excitation with atomic specificity and spatial resolution. This ability to track the time-evolving charge distribution has direct relevance to understanding excited state quenching or charge separation in light harvesting and DNA photoprotection. Femtosecond resolution hard x-ray scattering and crystallography provide powerful tools for tracking the nuclear evolution initiated by optical excitation. This has particular relevance to understanding how proteins control chemical transformations. Critical to all of these applications is the expanded photon energy range enabled by the LCLS-II: significantly sub- $\AA$ wavelengths for high-resolution nuclear structure determination and long-wavelength soft x-rays for C, N, and O K-edge spectroscopy studies of electron dynamics. 


\subsubsection{Determining the Design Rules Used by Proteins to Control Chem- ical Transformations}

Biological systems have harnessed photo-induced charge transfer in photosynthesis and photo-induced isomerization in bacteriorhodopsin to fuel life. Biological systems also use photo-induced charge transfer and photoisomerization to control circadian rhythms, to see, to repair DNA, and to trigger motion [76-81]. Such systems provide clear demonstrations of effective light conversion for a wide range of biological processes. By understanding, mimicking, and manipulating these ultrafast processes, we have the opportunity to identify both molecular and supramolecular design rules for constructing artificial light-conversion materials and devices.

Photo-isomerization reactions in bacteriorhodopsin highlight the current limits of our present understanding of how a protein environment controls chemical reactivity. Ultrafast spectroscopy measurements have demonstrated that photo-isomerization of the retinal chromophore differs significantly from photo-isomerization in solution $[79,82]$. Ultrafast spectroscopy studies on bacteriorhodopsin mutants also demonstrate that modification of the protein environment significantly influences the photoisomerization dynamics and reaction pathway. While biochemical synthesis and ultrafast optical measurements have a demonstrated ability to influence and observe the outcome of photon-driven chemistry, these studies have not been able to explain these observations or identify the physical and chemical properties of the protein environment that dictate the outcome of photochemical reactions. Understanding how proteins control chemical reactivity requires time-resolved studies of the structure of both the isomerizing chromophore and the protein environment.

Serial protein nanocrystallography with ultrafast optical laser capability makes such experiments possible. The hard x-ray capabilities of LCLS-II provide the high intensity and short pulse length needed to carry out these studies. These measurements take specific advantage of an optimized nanocrystallography capability because of the intrinsic difficulties of the time-resolved crystallography and the need to make the measurements on multiple mutants and multiple protein systems to enhance the probability of success. These measurements will provide sufficient detail to constrain and benchmark quantum chemical dynamics simulations and enable the discovery of design rules for supramolecular chemistry.

\subsubsection{Converting Light to Fuel and Electricity Using Coordination Com- plexes}

The ability of inorganic complexes to catalyze a wide array of chemical reactions and strongly absorb visible radiation makes them targets for the development of artificial photosynthetic catalysts. Research in artificial photosynthesis uses natural photosynthesis as a template for designing materials and devices to efficiently and cost-effectively convert photons into fuel. Following light absorption, the first step in 
natural photosynthesis is the fast and efficient splitting of light-driven electronic excited states into charge-separated electrons and holes [83]. The effective design of an artificial photosynthetic reaction center must incorporate ultrafast charge separation and minimize the perturbation to the nuclear structure induced by the light-driven electronic excited state to inhibit undesirable photochemical and photophysical relaxation channels. Consequently, the ability to understand and eventually design molecular systems for solar applications requires a detailed understanding of electronic excited-state chemical dynamics and the molecular properties that govern them.

Scientists have designed and synthesized a wide variety of inorganic complexes that can generate charge-separated excited states, but ultrafast relaxation of the chargeseparated state greatly inhibits performance in many of these systems because a photocatalyst must reside in the excited state to drive a chemical conversion [84-86]. Controlling the excited-state dynamics of inorganic artificial photosynthetic systems first requires the characterization of the time-dependent charge distribution of the electron and hole and how this charge distribution influences the rate of electron excited-state relaxation. Soft x-ray spectroscopy, including x-ray absorption, fluorescence and inelastic x-ray scattering, provides a unique set of tools for tracking time-dependent changes in charge distribution of atomic specificity and Ångström resolution.

Understanding and eventually controlling molecular conversion of light to electricity and fuel in inorganic chemistry requires understanding the non-adiabatic dynamics of electronic excited states, which in turn requires understanding the coupled motion of electrons and nuclei. This proves more challenging in inorganic chemistry than in organic chemistry because of the myriad energetically accessible electronic spin states and stronger spin-orbit coupling in these systems. Self-seeded operations will also enable the simultaneous measurement of resonant inelastic and anomalous elastic x-ray scattering, a tour de force measurement currently unachievable at LCLS that the LUSI-II instrumentation will facilitate. We will also have the ability to measure time-dependent changes in the charge distribution with x-ray scattering and soft $\mathrm{x}$ ray spectroscopy utilizing the new LUSI-II instruments in both the soft and hard $\mathrm{x}$-ray regions. This allows us to investigate the charge separation dynamics with atomic resolution and specificity.

\subsubsection{Elucidating the Mechanism of Photosynthetic Water Splitting}

Over $90 \%$ of the energy storage in photosynthesis results from splitting two water molecules into molecular oxygen. In nature, this water oxidation reaction is accomplished effectively by the oxygen-evolving complex (OEC) in Photosystem II (PS II), a multi-subunit membrane protein of green plants, algae, and cyanobacteria. The OEC of PS II that catalyzes the reaction contains a heteronuclear $\mathrm{Mn}_{4} \mathrm{Ca}$ cluster that couples the four-electron oxidation of water with the one-electron photochemistry at 
the PS II reaction center, by acting as the locus of charge accumulation. Energy from the absorption of successive photons drives the OEC through a series of five intermediate $S$-states (Figure 3.6a), representing the number of oxidizing equivalents stored, after which a spontaneous reaction occurs that releases $\mathrm{O}_{2}$ and reforms the $S_{0}$-state. Although many alternatives have been proposed, solar energy is by far the most abundant and inherently clean energy source. Among the several possible renewable energy substrates, water emerges as an ideal candidate due to its ubiquity and energy storage potential. However, effective methods for water splitting have proven elusive, in part due to difficulties in managing the overall four-electron, fourproton redox chemistry. Given the role of PS II in maintaining life and the future vision of a renewable energy economy, understanding the structure of the $\mathrm{Mn}_{4} \mathrm{Ca}$ catalyst and the mechanism of the water oxidation reaction is considered one of science's grand challenges.

The detailed chemistry involved in the OEC has emerged slowly, and much of importance remains to be learned. The structure of the $\mathrm{Mn}_{4} \mathrm{Ca}$ complex in the $S_{1}$ state is emerging from x-ray scattering and $\mathrm{x}$-ray spectroscopy studies, but much less is known about the structural changes in the $\mathrm{Mn}_{4} \mathrm{Ca}$ cluster through the catalytic cycle, which are critical for understanding the mechanism of oxygen evolution. The intermediate $S$-states of the OEC, $S_{0}$ through $S_{3}$, have been studied to varying degrees using biochemical and spectroscopic techniques. However, almost nothing is known about the putative $S_{4}$ state, although experimental results, UV/Vis, EPR and x-ray spectroscopy indicate that there are some kinetically resolvable intermediate states present during turnover.

There are many proposed mechanisms for the photosynthetic water oxidation reaction and the formation of the $\mathrm{O}-\mathrm{O}$ bond. The favored mechanisms for water oxidation by the OEC are (Figure 3.6b): A) reaction of a bridging/terminal Mn-oxo unit with predominant radical character with an oxo/hydroxo/water ligand of $\mathrm{Ca} / \mathrm{Mn}$ or an exogenous $\mathrm{H}_{2} \mathrm{O}$; B) nucleophilic attack on a $\mathrm{Mn}(\mathrm{V}) \equiv \mathrm{O}$ or $\mathrm{Mn}(\mathrm{IV})=\mathrm{O} \bullet$ terminal ligand by Ca-bound water or hydroxide; $\mathrm{C}$ ) reaction involving an end-on peroxo ligand to a single Mn center.

Recent results at the LCLS have shown that the probe-before-destroy method based on femtosecond XFEL pulses is in fact suited to study the undamaged structure of PS II at room temperature [33,40,87], finally opening the door for x-ray studies of the entire Kok cycle. The work uses a combination of x-ray diffraction (XRD) and $\mathrm{x}$-ray emission spectroscopy (XES) and is aimed at studying the geometric and electronic structure changes during each $S$-state transition of the catalytic $S$-state cycle (Figure 3.6a). These time-resolved experiments are carried out in a pumpprobe mode, where the Mn-OEC and other model systems are advanced with visible light laser pulses and the XRD, XES or x-ray absorption spectroscopy (XAS) will be performed in the $6-10 \mathrm{keV}$ range. The higher flux provided by LCLS-II will be critical, as it will enable: (1) the observation of weak valence-to-core transitions 


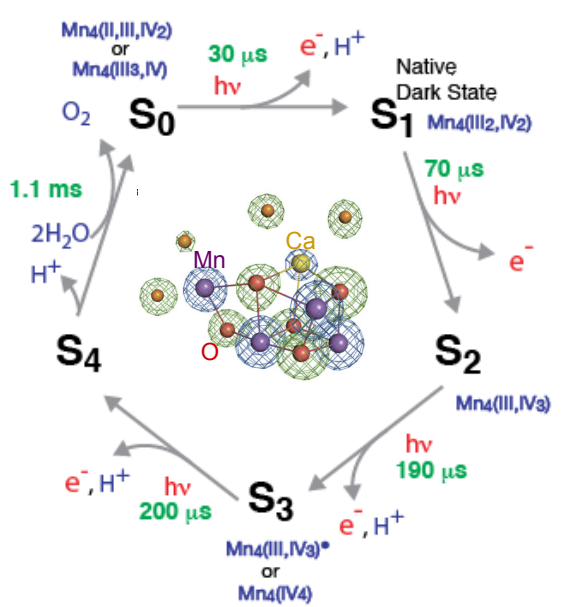

(a)

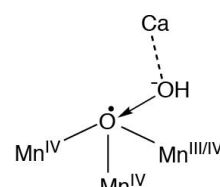

or

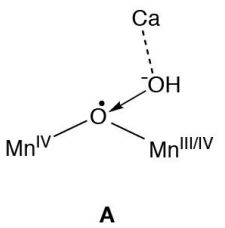

A

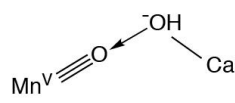

or

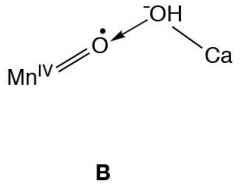

(b)

Figure 3.6: (a) The Kok $S$-state cycle for photosynthetic water oxidation and oxygen evolution from [89]. Inset - proposed structure from single-crystal polarized x-ray spectroscopy [90]. (b) Schematic showing three different proposals for the formation of the O-O bond during the $S_{3^{-}}\left[S_{4}\right]-S_{0}$ transition.

directly sensitive to the oxygen ligated to the Mn (these transitions are $\sim 100$ times weaker than Mn K $\beta$ XES (see [88])); (2) polarized XES spectroscopy to enhance the sensitivity of small structural changes; and (3) hard x-ray RIXS spectroscopy requiring a monochromatic x-ray beam to reveal more detailed information about the electronic structure changes, and thus reveal complementary electronic structure information. These studies will lead to a breakthrough in the understanding of photosynthetic water oxidation, in particular the formation of the critical O-O bond, which is likely to occur at the stage associated with the formation of the transient $S_{4}$ state.

\subsubsection{Understanding Nature's Photoprotection Mechanisms}

Deoxyribonucleic acid (DNA) carries our genetic information encoded in alternating base pairs, or nucleobases. Light-induced damage to the base pair sequence can eventually lead to cell mutations, resulting in cell death or cancer. The DNA in our skin is continually exposed to light, and the ultraviolet spectral region contains sufficient photon energy to damage DNA by either breaking the DNA double strand or fragmenting the nucleobases. The nucleobases, like many other heteroaromatic molecules, absorb in the near ultraviolet (UV) region transmitted by the atmosphere. The DNA is therefore exposed to constant photochemical stress by excitation of higher-lying electronic states. To avoid photochemical decomposition, the nucleobases have ultrafast pathways for funneling the population from the excited electronic state into the ground state via non-adiabatic dynamics. Just as for the inorganic complexes involved in artificial photosynthesis, it is important to be able to understand non-adiabatic coupling in nucleobases, although in this case it serves 
as a photoprotection mechanism rather than a light-harvesting mechanism.

Our current understanding of the photoprotection mechanisms in nucleobases is based largely on theoretical studies paired with ultrafast absorption and valence shell ion/photoelectron spectroscopy [91-94]. An example of the ultrafast relaxation mechanism for the nucleobase thymine is shown in Figure 3.7. The interpretation of the transient states is complicated, and the relaxation from the excited state to the ground state may only be possible with a violation of the Born-Oppenheimer approximation (BOA). Despite extensive theoretical and experimental efforts, the relaxation mechanism remains a matter of debate [91,95-97].

LCLS-II provides an opportunity to use ultrafast soft x-ray pulses to learn about nature's photoprotection mechanisms. Extremely short pulses, down to a single femtosecond in duration, provide a precision tool for probing non-BOA dynamics, complementing existing ultrafast spectroscopy methods. This will allow us to follow the electronic change as the nuclei move. In addition, at this timescale a new window opens: We will have the opportunity to follow pure electronic dynamics with motion periods on the order of attoseconds to a few femtoseconds [98,99]. The LUSI-II instruments proposed here, combined with soft x-ray self-seeding at LCLS-II, will deliver high-intensity, monochromatic pulses or two-color pulses with a well-determined and stable spectrum from shot to shot. This capability will enable stimulated emission and Raman transitions to probe, and possibly excite, the molecular valence electrons with picometer spatial fidelity at the SXT instrument. 


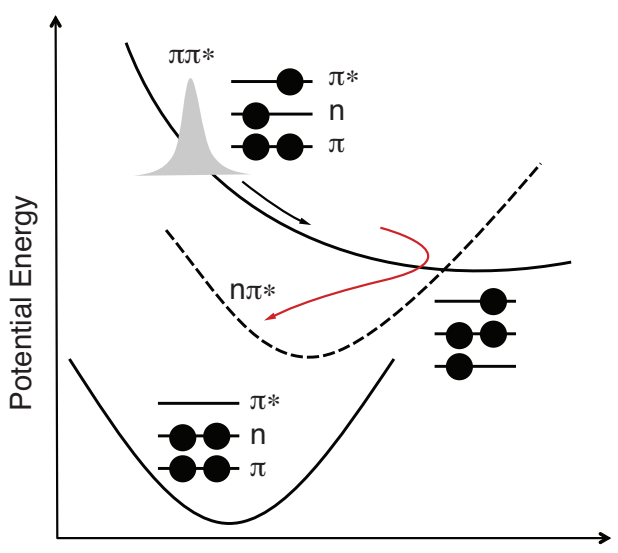

Effective Nuclear Path

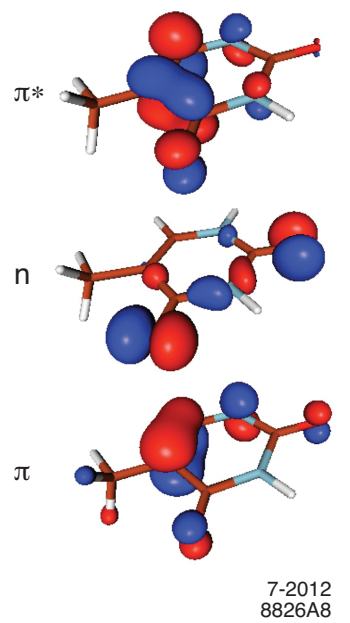

Figure 3.7: Suggested photoprotection mechanism of the nucleobase thymine. Molecular potential energy surfaces with orbital populations are on the right, and orbital geometries are plotted on the left. The ultraviolet light promotes an electron from the doubly filled $\pi$ orbital into the initially unoccupied $\pi^{*}$ state. In this so-called $\pi \pi^{*}$ state, the molecule is highly reactive with its environment. To counteract photo-induced reactions, the electronic energy is funneled into a lower-lying $n \pi^{*}$ state via non-adiabatic dynamics, as shown by the red arrow. 


\subsection{De novo Structure Determination of Challeng- ing Biological Targets}

When selecting the determination of the complex structure of a protein associated with African sleeping sickness as one of the nine runners-up for the 2012 "Breakthrough of the Year," the Science article states that the success at LCLS "shows the potential of x-ray lasers to decipher proteins that conventional x-ray sources cannot." Solving radiation-damage-free structures of challenging and important proteins and complexes that cannot be solved by synchrotron-based methods will require independent, de novo structure determination using single-wavelength anomalous dispersion (SAD) and multi-wavelength anomalous dispersion (MAD) methods with highly accurate intensity measurements. The enhanced energy range (to reach the Se and $\mathrm{Br}$ K edges), spectral intensity, and pulse control of LCLS-II, as well as the increased capacity, will be essential in accomplishing this. We will use two very challenging and high-impact research areas, HIV vaccines and neurodegenerative diseases, to illustrate the need for enhanced LCLS-II capabilities and the proposed NXD instrument.

\subsubsection{HIV Vaccines}

Developing effective HIV vaccines is one of the biggest challenges in human health. HIV-1 Envelope (Env) trimers on the surface of HIV are considered one of the most appropriate targets, but so far only low-resolution EM structures are available (see Figure 3.8). A number of research groups around the world are intensively working on crystal structures of the HIV Env trimers, but crystals do not diffract to high enough resolution even at the brightest synchrotron beamlines. Hence, the HIV-1 Envelope trimer structure has been identified as one of the top five most important crystal structures to be solved in the biological sciences [100].
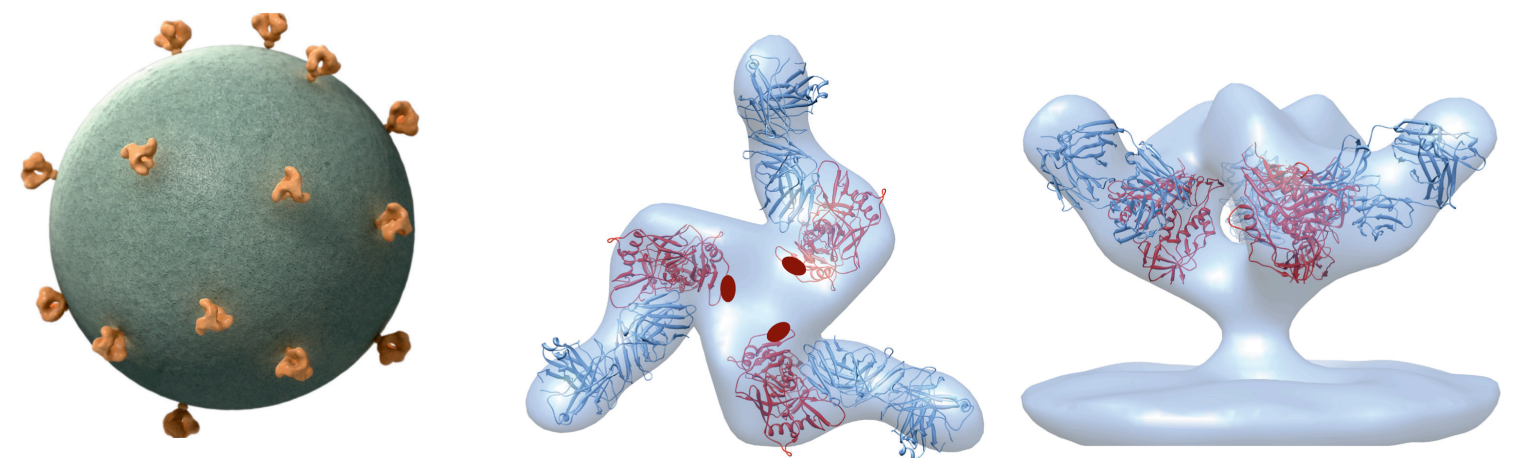

Figure 3.8: Trimeric Env spikes displayed on the surface of HIV (left). HIV electron density obtained from cryo-EM with models of the the HIV-1 Envelope trimers [101]. 

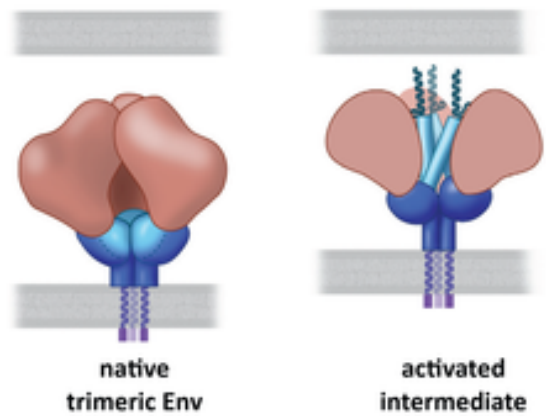

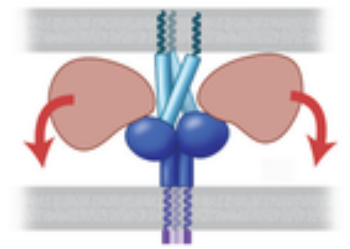

pre-hairpin intermediate

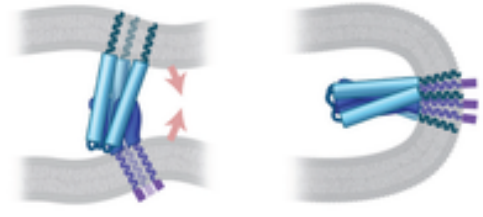

six-helix bundle formation/ membrane fusion

Figure 3.9: Entry of the HIV particle into the human host cell. Figure taken from [101].

The Env trimer, shown in Figure 3.9, has two components - gp120 (brown) and gp41 (blue). Env needs to be activated by interaction with the human host cell, CD40, and/or co-recepter (not shown in Figure 3.9), which triggers a large conformational change of the trimer to "pre-hairpin intermediate" (middle panel). After this, three gp120 molecules dissociate from the complex, leaving gp41 to bring two membranes (HIV, bottom and human host cell, above) close to each other, eventually fusing the two membranes into one (right-most panel), accomplishing the entry of the HIV particle into the human host T4 cell.

Successful development of vaccines against the Env trimer will help inhibit the fusion process between HIV particles and human host cells. The protein complexes depicted in Figure 3.9 undergo large-scale conformational changes during the fusion process. For example, the combination of gp120 conformational flexibility, sequence variability, and extensive glycosylation enables the protein to constantly evade the immune system and, by the same token, has rendered this molecule particularly difficult to crystallize, and the resulting crystals are extremely small and radiation sensitive. Higher-energy and better-controlled LCLS-II beams will allow radiation-damagefree de novo structure determination of these complexes to help the development of effective vaccines against HIV.

\subsubsection{Controlling Neurodegenerative Diseases}

Neurodegenerative diseases, such as Alzheimer's and Parkinson's diseases, exhibit misfolded proteins and peptides forming partially folded aggregates within the cell. It has been very challenging to obtain crystals large enough for structure determination using synchrotron radiation. Microfocus synchrotron undulator x-ray beams were indispensable for structure determination of amyloids from microcrystals (see Figure 3.10) [102, 103]. Researchers, however, are experiencing limitations with the state-of-the-art SR microfocus beamlines when pushing the sizes of amyloid crystals to the nanometer scale. An eventual goal of these studies is to develop drugs to control amyloid formation in the cells of patients with neurodegenerative diseases, and 


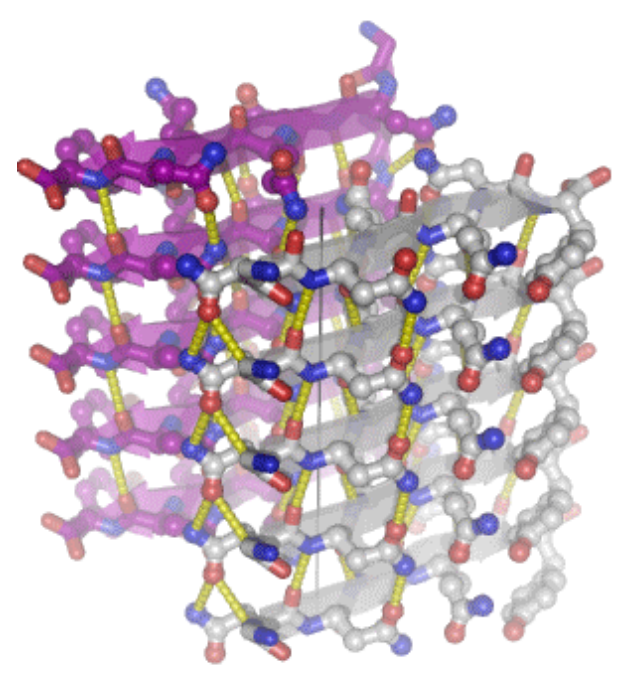

(a)

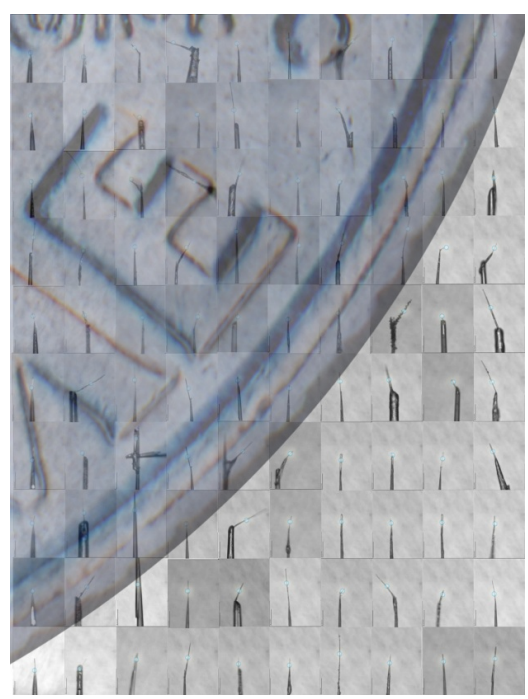

(b)

Figure 3.10: A crystal structure (a) and a montage of 100 microcrystals (b) used to determine the x-ray structures of amyloid fibril cross-beta spines. Each small circle indicates where the x-ray beam intersects that crystal. To provide scale, an image of a U.S. dime is superimposed. Credit: Michael Sawaya, David Eisenberg/UCLA. Shown by David Eisenberg during the LCLS-II New Instruments Workshop. Source: http://www.esrf.eu/news/general-old/general2007/disease/image/image_view_fullscreen.

understanding when and where the fibril formation occurs and propagates within the cell is of fundamental importance. LCLS-II will greatly enhance the ability to study the amyloid formation process within the cell by in situ analysis of crystalline aggregates, due to the ability to utilize smaller crystals than ever on a dedicated crystallography end station.

\subsubsection{New Capabilities Enabled by LCLS-II}

All of the protein structures published from data collected at LCLS thus far have been determined using the molecular replacement method for phase determination. This method, although very powerful, has inherent limitations due to the need for suitable search models. For determination of completely new and challenging structures, or structures with significant conformational changes compared to the search models, one generally needs to determine phases experimentally. But the spiky nature and shot-to-shot variation of the SASE photon energy spectra have so far prevented extraction of intensity measurements at the accuracy needed for de novo phase determination. The accuracy one can obtain from the "Monte Carlo Integration" approach, used in the first protein crystallography experiments at LCLS [31] for data reduction is, at this time, not known to be sufficient for this purpose. Several 
groups around the world, including the collaborators on this proposal, are developing methods to incorporate photon-energy spectra measurements into the data analysis packages for more accurate reflection partiality estimates. Nonetheless, it could be much more advantageous to utilize x-ray pulses with smoother and better defined photon energy spectra, which could enable intensity measurements sufficiently accurate for de novo phasing and more accurate intensities at LCLS-II.

Developing optimized crystallographic instrumentation and sample delivery are necessary for developing a robust de novo structure determination method at LCLS but not sufficient. As with the MAD and SAD methods at storage rings, accurate data is critical. This will require further developments of the photon source as well. Wellcontrolled photon pulses for most accurate spectral intensity measurements with a maximum number of fully recorded reflections will minimize the need for high redundancy in the measurement and thus large sample quantities, which has been an important limitation for proteins that are difficult to prepare and crystallize. There are intriguing developments that show promise to provide control of the wavelength distribution of the LCLS x-ray beam that the variable-gap undulators of LCLS-II could make routine. Recent LCLS accelerator developments have demonstrated several methods to produce two-color spectra - for example, by using a chicane and two sets of undulators [104]. There are also recent initial studies of improved SASE (iSASE) mode, which is based on the introduction of a set of delays in the electron beam along the undulators. By modifying the undulator parameters, this mode can produce an energy spectrum with a very sharp peak, near the transform limit, with a stable pulse intensity. Recent LCLS source development experiments of the iSASE have clearly demonstrated that one can produce simultaneous two-color LCLS XFEL pulses by setting the undulators in two groups with different resonant wavelengths. This method will allow the generation of two simultaneous pulses with different energies and with a fixed energy separation up to $2 \%$, i.e., 80 to $160 \mathrm{eV}$ for $8 \mathrm{keV}$ photons, between the two maxima, and a photon energy spectrum smoother than that from a SASE spectrum. The resulting energy spectrum of the two-color generation is substantially smoother than that of the conventional SASE or even iSASE spectra. The widths of the two peaks are around $5 \mathrm{eV}$, or about $0.1 \%$ bandwidth. These developments are encouraging and will continue in parallel with developments on sample delivery leading to an optimized nanocrystallography capability at LCLS.

In many cases $\mathrm{x}$-ray energies above $10 \mathrm{keV}$ are needed, as they are critical for accessing the important $\mathrm{K}$ edges for phasing such as $\mathrm{Se}(12.7 \mathrm{keV})$ and $\mathrm{Br}(13.5 \mathrm{keV})$. Higher energy x-ray photons up to $18 \mathrm{keV}$ will enlarge the sampling volume in the reciprocal space. In fact, experiences at the synchrotron sources show 12 to $18 \mathrm{keV}$ x-ray photons produce significantly higher resolution diffraction data compared to $8 \mathrm{keV}$ radiation owing to the diffraction geometry. Higher energy x-ray pulses of LCLSII will significantly increase the capability for recording highest-resolution data as compared to the current limit of LCLS, $10 \mathrm{keV}$. LCLS-II will be able to operate at these higher energies with enhanced pulse control and optimized crystal delivery sys- 
tems that will minimize sample consumption. We expect that applying the two-color mode to structural biology, using either the two photon energy peaks or the coalesced single peak at higher energies above $10 \mathrm{keV}$, would open up completely new ways to collect crystallographic diffraction data with LCLS-II: radiation-damage-free de novo structure determination using the MAD or SAD experimental phasing methods, and accurate data collection of fully recorded reflections. This should pave the way for development of a reliable de novo structure determination method using LCLS-II for challenging biological problems that cannot be tackled by the storage ring-based, high-brilliance light sources. 


\section{Instrumentation}

By providing two new FEL x-ray beams, LCLS-II will triple the total amount of user beam time at the LCLS. The LCLS-II project itself includes the beam transport, contained within the X-ray Experimental Systems element of LCLS-II, to one x-ray instrument in the soft x-ray photon energy range. The LCLS-II project beamline will provide a focused non-monochromatic beam for high-field atomic physics and imaging experiments. Additional x-ray instruments are needed to productively utilize the additional FEL capacity provided by LCLS-II.

Before describing the required facilities, it is useful to define some key terms. The term "beamline" describes an optical transport and manipulation system that delivers x-rays to an "end station" which may be exchangeable. An end station consists of equipment to introduce and manipulate samples, along with all the necessary detectors and spectrometers to examine particles and x-rays emitted from the sample, and may also incorporate special optics for additional manipulation, such as refocusing the incident beam. We shall use the term "instrument" to mean a complete user-ready facility that includes a beamline with all required optics and a completely integrated end station.

Figure 4.1 gives the overall layout of the LCLS-II experimental hall and indicates the potential location of up to three soft and three hard x-ray stations. Three soft x-ray instruments (SXT, SXU, SXM) and two hard x-ray instruments ( $\AA$ XE and NXD) are included in this proposal. The unoccupied location will be reserved for future instrumentation.

The soft x-ray instruments are located at the upstream end of the hall because of the larger divergence of the soft x-ray radiation compared with hard x-ray radiation. From our experience with soft x-ray operation at LCLS, hutch walls are not required for radiation shielding but barriers may be required for the containment of optical laser beams. The SXU beamline is part of the LCLS-II project scope; however, a new end station incorporating tight focusing optics and advanced detector systems is included in this proposal. The SXT instrument contains a grating monochromator and multi-spectroscopy end station for chemical reactivity studies. A high-resolution 


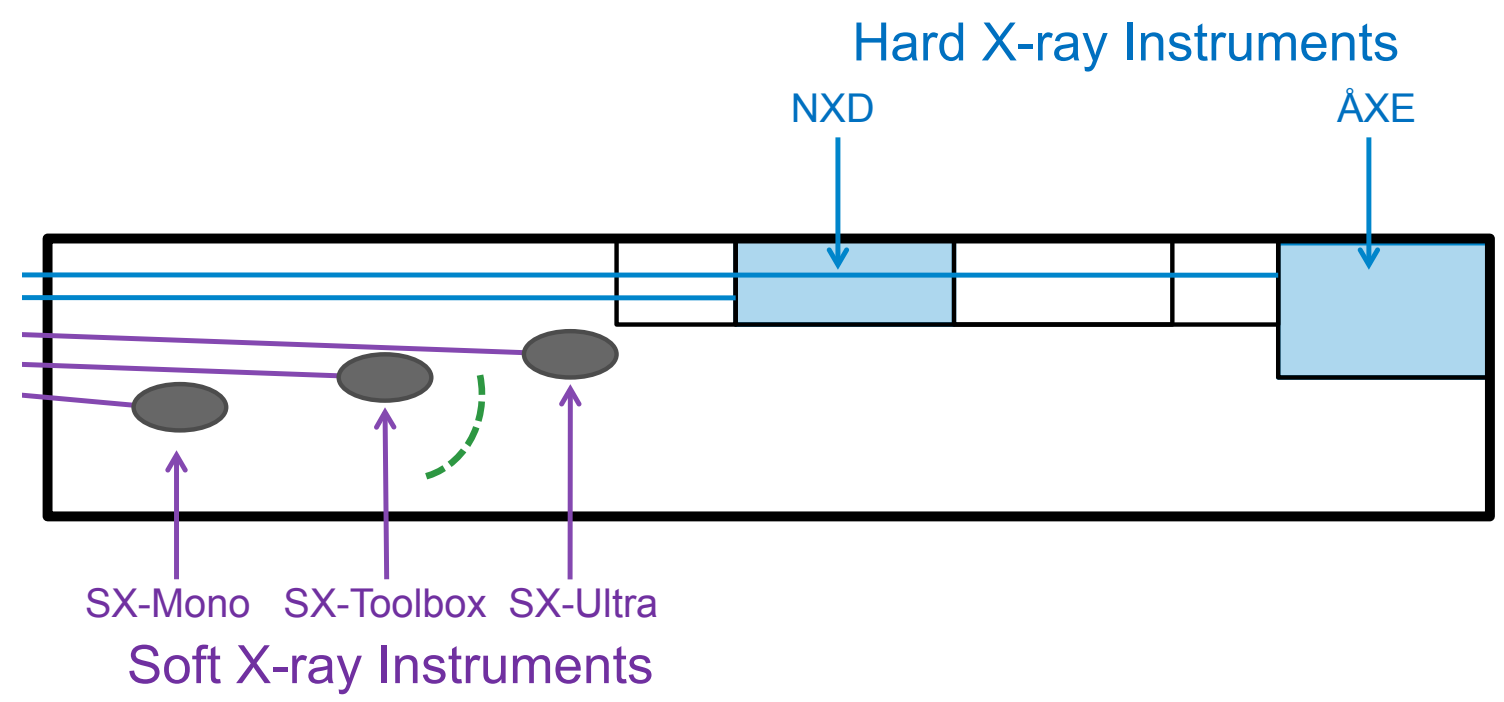

Figure 4.1: Overall layout of the x-ray instruments in the LCLS-II experimental hall.

resonant inelastic scattering (RIXS) end station, the extent of which is indicated by the dashed green arc in Figure 4.1, is also envisioned for the SXT instrument. The SXM beamline utilizes the grating monochromator of SXT but diverts the beam to a different interaction region with a retractable mirror system. A resonant scattering end station is envisioned for the SXM instrument.

The hard x-ray instruments are located at the downstream end of the LCLS-II hall. They need to be enclosed within radiation shielding hutches as indicated in Figure 4.1. The NXD instrument is dedicated to accurate structure determinations of macromolecules beyond radiation damage resolution limits. The general multipurpose hard x-ray instrument $(\AA \mathrm{XE})$ serves a variety of hard x-ray spectroscopy and elastic scattering experiments in materials science and chemistry. It combines the capabilities of the present x-ray pump probe (XPP) and x-ray correlation spectroscopy (XCS) instruments at LCLS. It is envisioned that the present XCS instrument will be converted in the future to a hard x-ray inelastic x-ray scattering (IXS) instrument. 


\section{1 Ångström X-ray Experiments ( $\mathrm{XXE})$ Instrument}

\section{Primary Science Opportunities Addressed}

- The role of complexity in materials properties (Section 3.1)

- Understanding and controlling the photochemical transformations on the molecular level (Section 3.2)

\subsubsection{Introduction}

The LCLS-II hard x-ray undulator will be a world-leading source when fully equipped. The scientific topics addressed by the $\AA$ XE instrument are nearly identical to those addressed by the SXT and SXM instruments: characterizing the behavior of photoexcited molecules and materials of technological importance. Hard x-ray probing techniques yield information complementary to that from soft $\mathrm{x}$-ray techniques. While soft x-ray tools are used primarily to characterize the electronic structure of a system, hard x-rays excel at directly measuring the atomic arrangements. As such, in many cases both probing techniques are needed to fully elucidate the fundamental mechanisms involved at the earliest stages of an ultrafast process due to the various couplings of electronic and atomic structure. In addition, hard x-rays have the ability to penetrate deep into materials and effectively probe bulk effects. This attribute is also advantageous when experiments involve sophisticated sample environments where entrance and exit windows are common. The AXE instrument will be designed to exploit these properties.

\subsubsection{Instrument Concept}

The AXE instrument will fully exploit all x-ray beam properties of the LCLS-II hard x-ray undulator: high brilliance on the first harmonic up to photon energies of $18 \mathrm{keV}$, short pulse duration, narrow self-seeded bandwidth, and near-full transverse coherence. The AXE instrument design will emphasize flexibility, ease of use, and rapid reconfiguration capability. This instrument will accommodate a wide variety of sample environments (in-air, vacuum, cryogenic temperatures, high pressure, etc.) and will execute a wide variety of x-ray probing techniques (WAXS, SAXS, XAS, XES, XPCS, CXDI). In many instances, AXE will be capable of performing simultaneous combinations of the aforementioned x-ray probing techniques.

The majority of ÅX experiments will involve the creation and probing of nonequilibrium states of molecular and condensed matter systems. These states typically will be generated with an optical laser. As such, a complete ultrafast optical laser system is included in the design of the $\AA$ XE instrument. This system contains wavelength conversion capabilities to populate specific degrees of freedom within a 


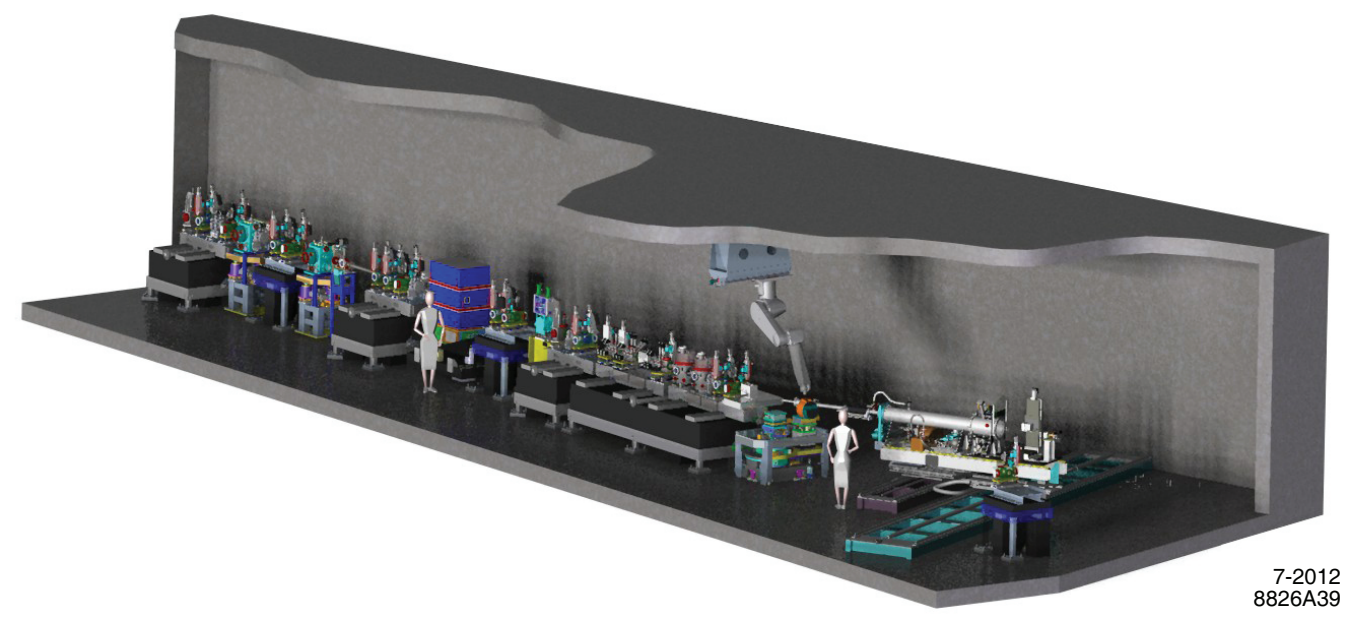

Figure 4.2: Rendering of the proposed multipurpose hard x-ray instrument $\AA$ XE at LCLS-II.

sample, a timing system capable of achieving a $\sim 10$ fs resolution, and amplification stages to reach the desired level of excitation densities. AXE will also have the capability of performing x-ray pump/x-ray probe experiments via a split-and-delay system.

\subsubsection{Specifications}

The AXE instrument will operate in the $5-18 \mathrm{keV}$ photon energy range, with the majority of use expected in the higher end of the range. The lower energy cutoff is limited by the expected operational limit of the monochromator system. The upper limit is defined by the mirror distribution system energy cutoff. An inline focusing system, based upon refractive lenses, will be used to create a variable $\mathrm{x}$-ray spot size on the sample. The system will be capable of generating spot sizes from the unfocused beam size down to 2 microns. The AXE monochromator is an inline system that can be retracted. As such, the x-ray bandwidth can be tuned from the natural SASE bandwidth of $10^{-3}$ to either $10^{-4}$ or $10^{-5}$ via $\mathrm{Si}(111)$ or $\mathrm{Si}$ (333) operation of the $\AA \mathrm{XE}$ monochromator respectively. Table 4.1 shows the specifications for the $\AA \mathrm{XE}$ instrument.

\subsubsection{Role in the LCLS Complex}

Third-generation synchrotron user facilities, such as the Advanced Photon Source at Argonne National Laboratory, typically have more than 30 beamlines with each beamline containing multiple experimental stations. These facilities have the ability to construct many specific, purpose-built instruments due to the sheer quantity of instrument locations available. LCLS, on the other hand, has a very limited number of experimental stations for a given undulator source. This limitation, in combination with our observation of a rapidly evolution of scientific scope being addressed by LCLS-I, makes it important to construct at least one flexible, general purpose 
Table 4.1: Specifications for the ÅXE instrument.

\begin{tabular}{|l|l|}
\hline \hline & $\AA \mathrm{XE}$ \\
\hline Photon energy range & $5-18 \mathrm{keV}$ \\
Bandwidth & $10^{-3}$ to $10^{-5}$ \\
Focusing optics & Refractive lenses \\
Spot size & $2-300 \mu \mathrm{m}$ \\
Monochromator & Yes \\
Sample environment & Reconfigurable diffractometer system \\
Detector needs (high signal) & $3000 \mathrm{x} 3000$ pixels \\
& $100 \mu \mathrm{m}$ pixel size \\
& 10,000 dynamic range \\
Detector needs (low signal) & $3000 x 3000$ pixels \\
& $50 \mu \mathrm{m}$ pixel size \\
Optical laser & Low noise \\
& $20-300$ fs pulse duration \\
\hline
\end{tabular}

instrument for each unique undulator source at the LCLS complex. AXE fulfills this roll at the LCLS-II hard x-ray undulator source and will be the catch-all instrument capable of performing experiments that are not feasible on the purpose-built NXD instrument.

$\AA \mathrm{XE}$ is analogous to the multipurpose LCLS-I XPP instrument, one of the most-indemand instruments at LCLS to date. Although there is some overlap between these instruments, there are many noteworthy differences:

- AXE will typically run at higher photon energies, since the LCLS-II hard x-ray source will be capable of self-seeding at photon energies up to $18 \mathrm{keV}$. The LCLSI source is currently limited to $10 \mathrm{keV}$ and does not have the undulator length required to saturate the self-seeded beam at high photon energies.

- AXE will be equipped with an integrated two-theta arm on the diffactometer system, which will be ideal for studies requiring crystal energy analyzers.

- AXE will utilize a 4 meter horizontal detector arm that is capable of reaching a 90 degree scattering angle

- ÅXE will contain a x-ray split-and-delay system 


\subsection{Soft X-ray Toolbox (SXT) Instrument}

\section{Primary Science Opportunities Addressed}

- The role of complexity in materials properties (Section 3.1)

- Understanding and controlling the photochemical transformations on the molecular level (Section 3.2)

\subsubsection{Introduction}

LCLS-II provides a foundation to discover mechanisms necessary to develop functional materials and control chemical processes. Soft x-rays access these mechanisms through an understanding of electronic structure and order with elemental and chemical specificity. The soft x-ray toolbox instrument will deliver unique experimental access to femtosecond-timescale electron dynamics via advanced spectroscopic techniques (time-resolved resonant inelastic x-ray scattering, stimulated scattering). This is achieved by a new ability to deliver, control, and detect precision x-ray pulses.

\subsubsection{Instrument Concept}

The advanced spectroscopy methods the SXT instrument will utilize require independent temporal and spatial control of x-ray pulses in a way that is only available at optical wavelengths. To achieve this control with x-ray pulses, a configurable grating monochromator will be incorporated into the SXT instrument. This monochromator will be shared with the SXM instrument discussed later in this chapter. Figure 4.3 displays a schematic layout of the SXT instrument, where a highly configurable set of x-ray gratings, mirrors, and slits is used to create an "x-ray optical toolbox." The toolbox takes advantage of the monochromator and focusing mirror augmented by additional mirrors and slits that provide a flexibility in configuration approaching conventional laser optomechanics.

A resonant spectroscopy end station is proposed for SXT. Two dedicated sample environment systems are envisioned: a UHV system for surface science and catalysis and a liquid jet injection system operating at higher pressures for chemical dynamics occurring in solution. This combination of beamline and end station provides the perfect development platform for advances in nonlinear spectroscopies that will become standard techniques at soft x-ray FELs far into the future.

A high-resolution soft x-ray resonant inelastic x-ray scattering (RIXS) spectrometer will be constructed on SXT. This spectrometer will allow elementary excitations in many types of complex materials to be resolved through the observation of energy, momentum, and polarization dependence of x-ray emission with emphasis on lowenergy charge, spin, orbital, and lattice excitations of solids. It will be designed 


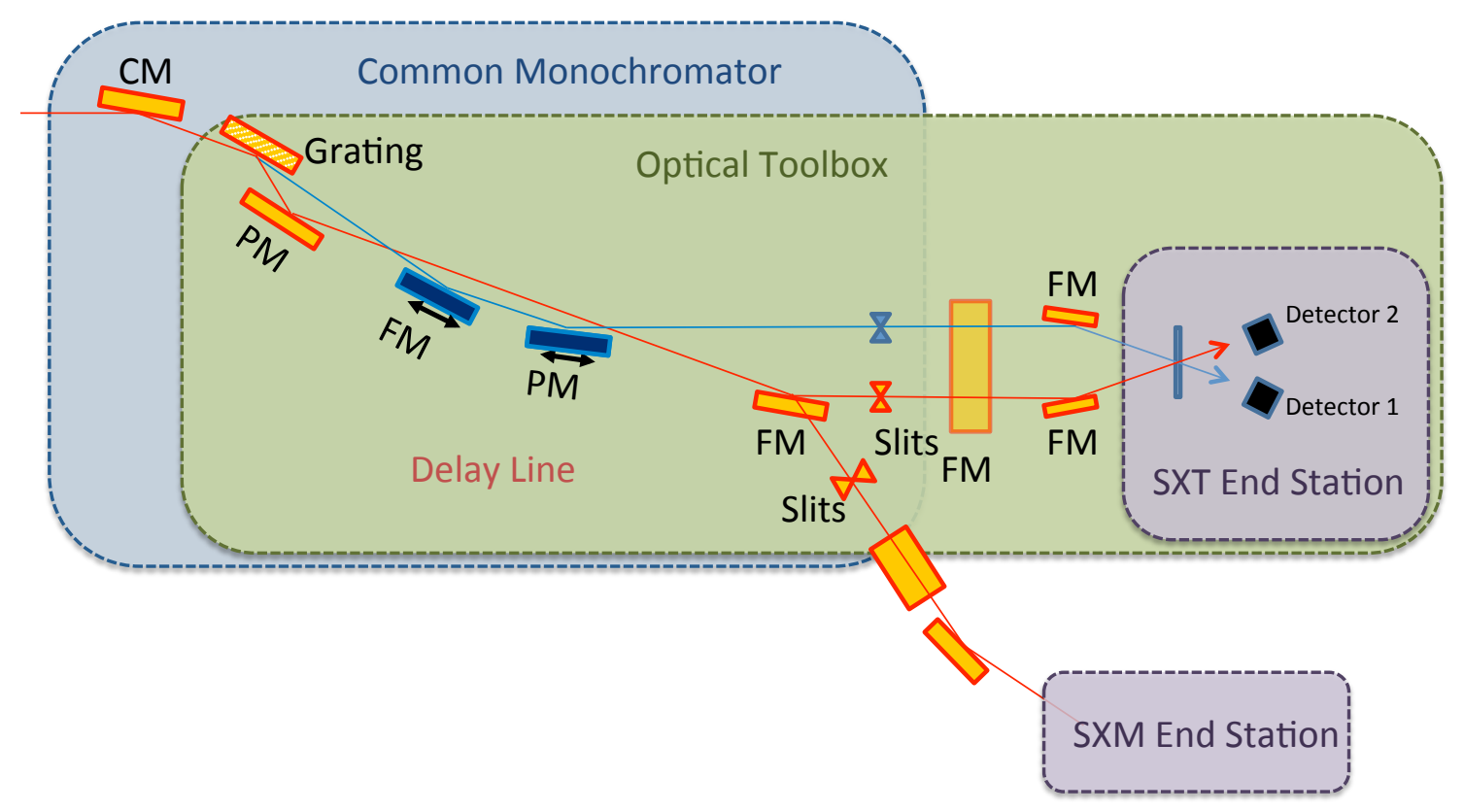

Figure 4.3: Schematic layout of the SXT and SXM optics. [CM: Collimating Mirror, FM: Focusing Mirror, PM: Plane Mirror]

to resolve fine energy features at moderate time resolution. It will also allow timeresolved RIXS by pump-probe techniques. The goal for this station is to be able to perform at a resolving power near 30,000 and cover a range of 400 to $1700 \mathrm{eV}$ photon energy. This would give $30 \mathrm{meV}$ energy resolution, which would be state of the art. Pulse broadening limits the possible time resolution to 70-300 fs at this spectral resolution, depending on the photon energy.

One important point to consider for spectroscopy development is the damage threshold of materials at the fluences available at LCLS-II. Currently, for instance, solid samples can handle a typical spot size of about $300 \mu \mathrm{m}^{2}$ with minimal attenuation for $3 \mathrm{~mJ}$ and $1 \mathrm{eV}$ bandwidth pulses. Damage concerns are ameliorated by using a line focus in the dispersive direction, such that the overall fluence is preserved. For instance, considering a spot size of $6 \mu \mathrm{m}$, focused to $300 \mu \mathrm{m}$ in the orthogonal direction, and a $7 \mathrm{~m}$ spectrometer (a length compatible with the layout of the LCLSII experimental hall), preliminary calculations give a resolution within a factor of two of the goal. Further optical design work will optimize the monochromator and spectrometer performance. The size of this instrument will necessitate a permanent installation at LCLS-II.

In combination with the x-ray optical toolbox, RIXS studies can also be carried out in a stimulated Raman scattering geometry. The use of two pulses that traverse the sample at different angles has two great advantages. First, it provides stimulated gain in the direction of the second pulse and second, the stimulated beam is directed into the detector and therefore gives a large gain of solid acceptance angle. Enhancements 
by many orders of magnitude are possible, which helps circumvent electronic damage.

\subsubsection{Specifications}

X-ray emission and RIXS will be the primary techniques employed on SXT. Emission spectra will be measured using two dedicated varied line spacing plane grating spectrometers attached to the sample environment chamber. The spectrometers will utilize different gratings for optimal operation over a larger energy spread, but can also operate using the same grating to double the signal level. A full frame rate $(120 \mathrm{~Hz})$ soft x-ray sensitive area detector will be collaboratively developed for use on these spectrometers. The specifications for the SXT instrument are given in Table 4.2 .

Table 4.2: Specifications for the SXT instrument.

\begin{tabular}{|l|l|}
\hline \hline & SXT \\
\hline Photon energy range & $250-1800 \mathrm{eV}$ \\
Optics & Tool kit, KB focusing mirrors \\
Spot size & $5-2,000 \mu \mathrm{m}$ \\
Monochromator & Yes \\
Resolving power & $1,000-30,000$ \\
Primary end station & Multi-spectroscopy \\
Main detector & X-ray grating spectrometer \\
Detector needs & $1000 x 1000$ pixels \\
& $10 \mu \mathrm{m}$ pixel size \\
& $120 \mathrm{~Hz}$ \\
Optical laser & Low noise \\
& $20-300$ fs pulse duration \\
\hline \hline
\end{tabular}

\subsubsection{Role in the LCLS Complex}

The SXT instrument will be dedicated to advanced soft x-ray spectroscopic techniques such as XES, RIXS and stimulated RIXS. It will utilize various optics to precisely manipulate and control the FEL pulses produced at LCLS-II. 


\subsection{Nanocrystal X-ray Diffraction (NXD) Instrument}

\section{Primary Science Opportunity Addressed}

- De novo structure determination of challenging biological targets (Section 3.3)

\subsubsection{Introduction}

The use of micron- and sub-micron-sized crystals of protein macromolecules for structure determination has been an early success for LCLS-I and is expected to continue as a high-demand research area in the coming years, in direct analogy to the evolution of macromolecular crystallography at third-generation x-ray user facilities. The instrumentation required to deliver a fully optimized Nanocrystal X-ray Diffraction (NXD) instrument is described in this subsection. NXD will provide a purpose-built small-crystal facility at LCLS-II to the general user community. The benefits of a dedicated facility include the opportunity to provide a stable foundation of LCLSproven components and provide a workhorse for protein structure determination. This instrument complements the CXI instrument at LCLS-I by providing a highthroughput backdrop against which high-risk, extraordinary-outcome research can be performed at the more flexible CXI instrument, which will continue to provide tighter focusing necessary for the work towards single molecule imaging using hard $\mathrm{x}$-rays.

In order to fully utilize the potential of LCLS-II for protein nanocrystallography, the NXD design will accommodate higher photon energies (up to $16 \mathrm{keV}$ ) for molecular systems requiring higher resolution and a larger energy bandwidth by operating in the "over-saturation" regime of the FEL. This will allow the exploitation of the selenium K-edge, with some margin, for Multi-wavelength Anomalous Dispersion (MAD) phasing and to capture higher-resolution data sets arising from this higher photon energy.

\subsubsection{Instrument Concept}

Most small-crystal protein crystallography experiments at LCLS-I are currently performed using liquid-jet sample delivery systems that constantly replenish the sample at the interaction region. While we anticipate increasingly sample-efficient delivery technologies, we believe that these new technologies will share certain characteristics with the current liquid-jet method. In particular, the beam will continue to be minimally attenuated by the sample delivery system. This is highly desirable, as both the attenuation of the diffraction signal and the undesired scattering from the sample's protective environment, e.g., the buffer in which the sample is delivered in a liquid jet, are minimized. 


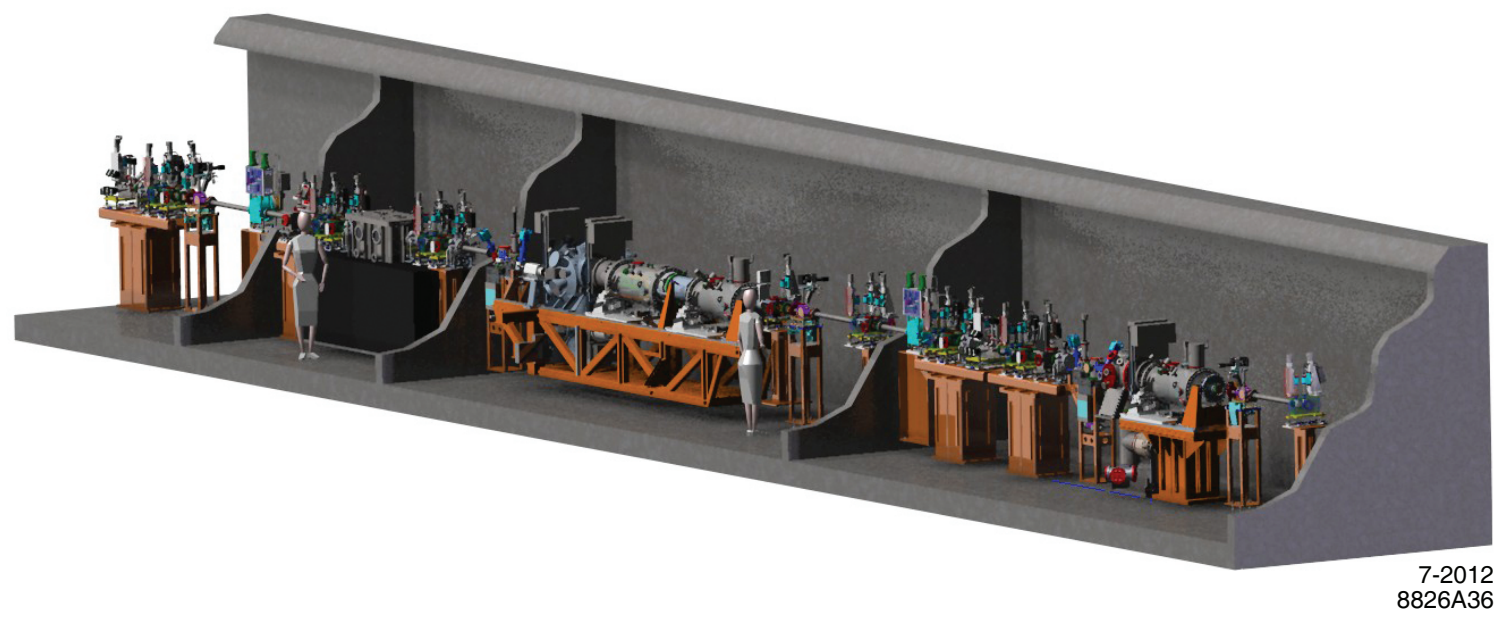

Figure 4.4: Rendering of the proposed Nanocrystal X-Ray Diffraction (NXD) suite at LCLS-II showing, from left to right, hutches containing LCLS-II common hard x-ray optics; an optics hutch containing KB focusing mirrors; the main experimental hutch (NXD) with sample environment, detectors and diagnostics; and finally the last hutch that utilizes the refocused beam (NXD-II).

The transmitted beam, after it passes through the hole in the middle of the 2-D diffraction detector, will typically contain close to $10^{11}$ photons per pulse with a nearly unchanged brightness. This "spoiled" or "spent" beam will be preserved to serve a future second end station downstream of the main instrument, in a separate hutch. This concept is beneficial on two key levels. First, the "spent" beam is not simply lost in a beam dump; rather the scheme will provide a way to reuse this beam and increase the capacity of LCLS-II. Secondly, it has been observed that classical x-ray sources, such as storage rings, are not adequate tools to determine the quality of small crystals or even to predict their diffraction quality at LCLS. The second hutch of the NXD suite will provide access to crystal screening non-invasively and make the use of the primary NXD hutch more efficient.

We call the primary hutch of the NXD suite "NXD." Here, the most challenging measurements requiring the highest power density, highest flux, and tight focusing will be performed. We envision that most pump-probe experiments requiring optical laser excitation will occur in NXD, and include that system in the NXD specification. The secondary instrument, "NXD-II", is located in a completely separate hutch to allow the configuration of and access to the current experiment on either end station with minimal effect on the other one. A rendering of the proposed NXD suite is shown in Figure 4.4 with, from left to right, front end components, an optics hutch, the NXD hutch, and the separate NXD-II hutch.

\subsubsection{Specifications}

The expected demand for the NXD instrument is for the study of small macromolecular crystals in the few-micron to sub-micron range where diffraction will be used to 
obtain a structure at the highest resolution possible and without radiation damage from the x-ray exposure. Therefore, the need for hard x-rays is clear. The higher energy limit available at the NXD instruments must at least reach the selenium Kedge to allow MAD phasing, as is typically done at crystallography beamlines at storage ring sources. The energy range must exceed the selenium edge by a margin of at least $2 \mathrm{keV}$ for proper MAD phasing and therefore a cutoff energy of $16 \mathrm{keV}$ is desired, which has the additional benefit of allowing higher-resolution measurements.

The lower energy cutoff is desired to be as low as possible within reasonable feasibility. The $16 \mathrm{keV}$ cutoff defines a maximum mirror angle which, in conjunction with the maximum reasonable mirror length afforded by current technology, limits the lowest usable photon energy to that at which the beam overfills the mirror apertures. We expect $6 \mathrm{keV}$ to be the lowest useable energy, although lower energies will still be transmitted with a loss to efficiency. Table 4.3 shows the specifications of NXD.

Table 4.3: Specifications for the NXD instrument.

\begin{tabular}{|l|l|}
\hline \hline & NXD \\
\hline Photon energy range & $6-16 \mathrm{keV}$ \\
Bandwidth & As wide as possible for SASE \\
Focusing optics & Well-defined and controlled for seeding \\
Spot size & Bendable KB mirrors \\
Sample environment & $0.5-3 \mu \mathrm{m}$ \\
Detector needs & Flexible experimental chamber \\
& $4000 x 4000$ pixels \\
& $100 \mu \mathrm{m}$ pixel size \\
Optical laser & 10,000 dynamic range \\
\hline \hline
\end{tabular}

\subsubsection{Role in the LCLS Complex}

The proposed NXD instrument will provide dedicated nanocrystallography capability that will greatly expand the capacity of the LCLS for crystallography. This new instrument will complement the existing CXI instrument by adding dedicated nanocrystal diffraction capacity, providing a stable, user-friendly instrument, and enabling higher-photon-energy diffraction experiments. CXI will remain available for nanocrystallography work, especially those studies requiring the smallest LCLS x-ray spots approaching $100 \mathrm{~nm}$. A dedicated nanocrystallography setup at LCLSII will provide a robust tool for structural studies while allowing more exploratory research using the existing CXI instrument to continue to push the limits towards single molecule imaging using hard x-rays. 


\subsection{Soft X-ray Ultra (SXU) Instrument}

\section{Primary Science Opportunities Addressed}

- Understanding and controlling the photochemical transformations on the molecular level (Section 3.2)

- De novo structure determination of challenging biological targets (Section 3.3)

\subsubsection{Introduction}

The ultra-high-intensity end station will serve the needs of experiments for which the highest possible photon density is required and the spectral contrast from the seeded source is sufficient, or for which a large bandwidth is advantageous. For biological imaging the general rule of thumb is that the experiments should be performed at the longest possible wavelength supporting the required resolution, as the scattering cross section decreases with increasing photon energies. The SXU end station will be optimized for delivering the maximum number of photons from the sulfur and phosphorous K-edges down into the water window between the carbon and oxygen edges. For the AMO community the SXU branch line will be a crucial advancement towards nonlinear x-ray physics. Laser-based research at the intensity frontier has led to many unexpected discoveries. Prominent examples in the optical regime range from high-harmonic generation and attosecond pulse creation to fusion in laser-driven cluster nanoplasmas. In the extreme nonlinear regime, electrons are driven nonperturbatively by the electric field of the laser pulse. Since the intensity required to access non-perturbative ionization scales inversely with the wavelength of the light, current x-ray free-electron lasers miss the non-perturbative regime by roughly two orders of magnitude in power density. LCLS-II, with its high peak output from the seeded soft x-ray undulator in combination with the SXU high-transmission $100 \mathrm{~nm}$ focus beamline, offers the potential to break into the nonlinear x-ray physics regime. Compared to the current state-of-the-art high-intensity x-ray beamline at LCLS, a factor of 10 increase in power density will result from the increased pulse energies available with the seeded source at LCLS-II along with an improved hightransmission beamline design with minimal optics. Another factor of 100 in intensity can be gained by focusing the beam down to $100 \mathrm{~nm}$ using specialized focusing optics. With these improvements the SXU beamline will deliver power densities exceeding $10^{21} \mathrm{~W} / \mathrm{cm}^{2}$ on target.

\subsubsection{Instrument Concept}

The beam transport from the source to the end station will be provided within the XES portion of the LCLS-II project. Within the LUSI-II project the basic beamline will be enhanced with ultimate focus optics delivering a $100 \mathrm{~nm}$ x-ray spot and a harmonic rejection system. To achieve the $100 \mathrm{~nm}$ focal spot, prefigured fixed focal length mirrors will be used. As the focal length will be short, the optics must be 
incorporated into the experimental end station. The end station will be designed as a modular system, allowing a variety of different experimental techniques to be employed with very tight x-ray focus. The core of the system will incorporate the focusing optics and interaction chamber, including multi-stage differential pumping sections between the two in order to protect the optics from the target samples. The interaction chamber will allow the incorporation of various sample delivery options and a wide range of particle spectrometers perpendicular to the beam. Pixelated charged-particle detectors will allow momentum-resolved multi-coincident electron and ion imaging experiments. Development of an integrated circuit chip with subnanosecond time resolution for such a detector is underway. Downstream of the interaction region will be a port for large-area photon detectors for imaging experiments. This modular, multipurpose system will allow for imaging and spectroscopy of dilute gas-phase targets.

\subsubsection{Specifications}

Table 4.4: Specifications for the SXU instrument.

\begin{tabular}{|l|l|}
\hline \hline & SXU \\
\hline Photon energy range & $250-2500 \mathrm{eV}$ \\
Focusing optics & KB focusing mirrors \\
Spot size & $100 \mathrm{~nm}$ \\
Monochromator & No \\
Primary end station & High-intensity imaging and spectroscopy \\
Main detector & Photon and particle pixel detectors \\
& Particle spectrometers \\
Detector needs & $1000 \mathrm{x} 1000$ pixels \\
& $10 \mu \mathrm{m}$ pixel size \\
& $<1 \mathrm{~ns}$ time resolution \\
Optical laser & $20-300$ fs pulse duration \\
& Variable wavelength \\
\hline \hline
\end{tabular}

\subsubsection{Role in the LCLS Complex}

The SXU instrument is designed for high-intensity applications and will thus be optimized for wide bandwidth beam applications in the soft x-ray spectral range. As such, this instrument will primarily perform experiments in the fields of nonlinear x-ray physics, high-field physics and nonperiodic imaging. 


\subsection{Soft X-ray Mono (SXM) Instrument}

\section{Primary Science Opportunities Addressed}

- The role of complexity in materials properties (Section 3.1)

- Understanding and controlling the photochemical transformations on the molecular level (Section 3.2)

\subsubsection{Introduction}

The Soft X-ray Mono instrument will leverage the existing infrastructure of the SXT instrument (grating monochromator) and provide a third beamline branch to perform experiments utilizing the soft x-ray undulator radiation of LCLS-II. SXM will enhance the soft x-ray science productivity at LCLS by permitting a two-on/oneoff instrument schedule arrangement, which is a much more efficient operating mode. In addition, SXM will focus on materials science experiments with the inclusion of a resonant diffraction end station proposed in the instrument scope.

\subsubsection{Instrument Concept}

Charge, spin, and orbital electronic order will be studied via resonant diffraction on SXM with a dedicated end station. A cryogoniometer is envisioned for this end station that can cool samples to $10 \mathrm{~K}$ with six-axis manipulator capabilities. The diffracted signal will be detected on a $120 \mathrm{~Hz}$ area detector sensitive to soft x-rays. In-vacuum motion of the detector about the Ewald sphere will provide access to sufficient momentum transfer. The specifications for the detector are given in Table 4.5 and it will be collaboratively developed. The small ( $\sim 10$ micrometer) pixel size will allow the detector to resolve coherent scattering speckles when the detector is moved several meters from the sample in an optional configuration. Image reconstruction will provide insight into the interplay between mesoscale order and electron and spin correlation. 


\subsubsection{Specifications}

Table 4.5: Specifications for the SXM instrument.

\begin{tabular}{|l|l|}
\hline \hline & SXM \\
\hline Photon energy range & $250-1800 \mathrm{eV}$ \\
Focusing optics & Bendable KB focusing mirrors \\
Spot size & $1-1,000 \mu \mathrm{m}$ \\
Monochromator & Yes \\
Resolving power & $1,000-30,000$ \\
Primary end station & Resonant scattering \\
Main detector & Articulated area detector \\
Detector needs & $1000 \mathrm{x} 1000$ pixels \\
& $\sim 10 \mu \mathrm{m}$ pixel size \\
& $120 \mathrm{~Hz}$ \\
Optical laser & $20-300 \mathrm{fs}$ pulse duration \\
& Variable wavelength \\
\hline \hline
\end{tabular}

\subsubsection{Role in the LCLS Complex}

The SXM instrument, like the SXT instrument, will utilize a monochromator system to control the spectral content of the soft x-ray pulses produced at LCLS-II. This instrument will be primarily dedicated to resonant diffraction experiments in the soft x-ray regime. 


\section{Potential enhancements}

\subsection{Nanocrystal X-ray Diffraction II (NXD-II) In- strument}

\subsubsection{Introduction}

The LCLS user community currently lacks a small-crystal screening facility. Such a facility would be of great benefit, particularly due to the observation that thirdgeneration x-ray facilities cannot reliably predict the quality of diffraction patterns observed from a given sample at the LCLS. The proposed downstream end station of the NXD suite (the NXD-II instrument) would provide an ideal opportunity to establish a small-crystal screening facility. The proposed NXD-II system has nearly all of the functionality of the main NXD hutch, allowing the transparent switch-over of a sample from the screening facility to the primary crystallography facility in the NXD hutch. While we foresee a high user demand for the screening facility, the downstream instrument could also be used for other forward scattering experiments, as long as these do not require the very highest flux density possible at LCLS-II. The NXD-II instrument would, for example, be an ideal location for studying larger crystals, mounted on a support of some kind, at atmospheric pressure and room temperature. Such measurements would be ideal for the common cases of metalloproteins, where resolution at a synchrotron is severely limited by radiation damage. The short pulses of LCLS-II could allow higher-resolution structural determination on these larger crystals.

\subsubsection{Instrument Concept}

The NXD-II instrument will utilize the beam that is left over after passing through the sample in the NXD hutch and passing through the holes in the NXD detectors. This minimally disturbed beam will then be refocused to the proper plane into the NXD-II hutch, where a fully functional nanocrystallography system similar to that in NXD, with similar sample environments, detectors, and diagnostics, will be available. A great example of the usefulness of NXD-II is the case of dynamic studies, where it 
would be very valuable to spend a few hours screening different sample preparation runs and selecting the best sample before attempting complex time-resolved measurements in the NXD hutch. This will greatly increase the efficiency of beam time use at LCLS-II.

\subsubsection{Specifications}

Table 5.1: Specifications for the NXD-II instrument.

\begin{tabular}{|l|l|}
\hline \hline & NXD-II \\
\hline Photon energy range & $6-16 \mathrm{keV}$ \\
Bandwidth & As wide as possible for SASE \\
& Well-defined and controlled for seeding \\
Focusing optics & Refractive lenses \\
Spot size & $1-10 \mu \mathrm{m}$ \\
Sample environment & Flexible experimental chamber \\
Detector needs & $4000 x 4000$ pixels \\
& $100 \mu \mathrm{m}$ pixel size \\
& 10,000 dynamic range \\
Optical laser & Yes \\
& $30-300 \mathrm{~ns}$ pulse duration \\
\hline \hline
\end{tabular}

\subsection{Hard X-ray IXS}

The implementation of the AXE instrument in the LCLS-II experimental hall not only increases the capacity and capabilities of the existing XPP instrument but also supports the user program of the XCS instrument located in the far hall of LCLS-I. The long scattering arm of the XCS instrument provides the opportunity to reconfigure this instrument for high-resolution inelastic x-ray scattering (IXS). It is envisioned that this type of spectroscopy has high potential when combined with the hard x-rays of LCLS. While IXS typically depends on average brightness, LCLS offers the opportunity for time-resolved studies while exceeding the average brightness of existing synchrotron radiation sources.

IXS at LCLS may revolutionize the field of hard x-ray inelastic experiments because of the unique properties of the LCLS hard x-ray undulator: high peak power, moderate repetition rate, small isotropic divergence and beam size, energy tunability 
of the first harmonic and, most importantly, the promise of fully saturated seeded pulses. A comprehensive review of the currently available IXS capabilities at thirdgeneration storage rings shows that operating IXS instruments can provide, on average, about $5 \times 10^{9} \mathrm{ph} / \mathrm{s} / \mathrm{meV}$ incident within the 5 to $25 \mathrm{keV}$ energy range. A fully saturated seeded LCLS is expected to deliver pulses with an average of at least 1 $\mathrm{x} 10^{12} \mathrm{ph} / \mathrm{s} / \mathrm{meV}$ over the same energy range. LCLS would therefore provide meV bandwidth pulses with a factor of 200 more flux than presently available. It would dramatically change the performance and scientific output of these photon-hungry experiments, due to the higher time-average brightness of LCLS. In addition, it would most importantly offer the unique opportunity to perform experiments in transient states, which is currently unavailable at any other facility.

Medium Energy Resolution (typically $0.1 \mathrm{eV}$ ) IXS is fundamentally different from High Energy Resolution (typically $1 \mathrm{meV}$ ) IXS from the instrumentation point of view. Therefore, two dedicated experimental setups are envisioned, with required parameters as listed in Table 5.2.

Table 5.2: Specifications for the IXS spectrometers on the XCS instrument.

\begin{tabular}{|l|l|l|}
\hline \hline & Medium resolution & High resolution \\
\hline Photon energy range & $5-15 \mathrm{keV}$ & $12-25 \mathrm{keV}$ \\
Bandwidth & $0.5 \mathrm{eV}$ & $0.5 \mathrm{eV} \mathrm{to}<1 \mathrm{meV}$ \\
Spot size & $50-100 \mu \mathrm{m}$ & $50-100 \mu \mathrm{m}$ \\
Monochromator & Yes & Yes \\
$\Delta E / E$ & $10^{-4}$ & $10^{-6}$ \\
Sample environment & Not critical & High pressure \\
& & High magnetic field \\
Detector needs & $50-100 \mu \mathrm{m}$ pixel size & $50-100 \mu \mathrm{m}$ pixel size \\
& $120 \mathrm{~Hz}$ & $120 \mathrm{~Hz}$ \\
& Low noise & Low noise \\
\hline \hline
\end{tabular}




\section{Management}

\subsection{Project Management}

LUSI-II project management will be organized in the LCLS Directorate of SLAC to ensure a close coupling of the project with the operating facility that will eventually assume the operations responsibility of the project deliverables. LCLS and SLAC staff will be assigned to the project from the departments with the core competencies necessary to design and construct the proposed LUSI-II instruments.

LUSI-II will employ project planning and earned value management systems now in place at SLAC. SLAC project management systems have been preserved and improved upon through the experience of many recent projects (LCLS, LUSI, MECI, FACET, LCLS-II). SLAC has a centralized project management group that supports the directorates with all aspects of project control and earned value management.

In addition to project management support, SLAC resources are available to provide procurement and Environment, Safety, Health and Quality (ESH\&Q) support to the LUSI-II project. Both divisions are staffed with experienced people to support this project during the planning and execution stages.

Scientific and engineering manpower requirements for LUSI-II will be significant and new hires will be recruited into the respective LCLS departments, from which the project will matrix effort to meet this demand. The LCLS Directorate is well equipped to take responsibility for LUSI-II construction due to the accumulating experience and expertise in operation and construction of LCLS instruments. As the LUSI-II project ramps down, the project staff will fold into the operations of the combined LCLS-I and LCLS-II facilities. 


\subsection{Organization}

The LUSI-II project will be managed within the LCLS Directorate at SLAC.

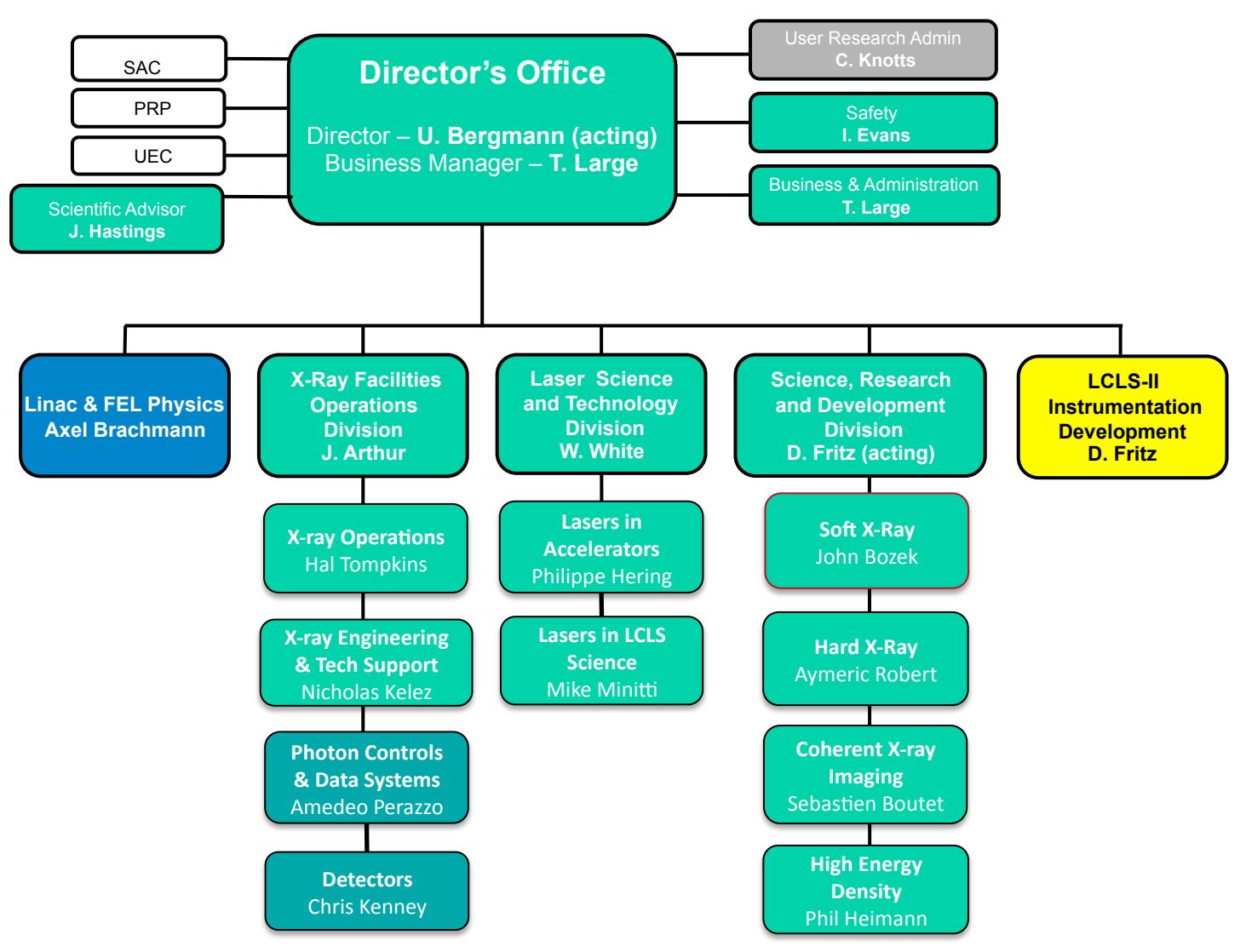




\subsection{Schedule}

Table 6.1: Major milestone schedule.

\begin{tabular}{|l|c|}
\hline \hline Milestone Event & Fiscal Year \\
\hline CD-0 Mission Need Approval & FY 2013 \\
CD-1 Approve Alternate Selection and Cost Range & FY 2015 \\
CD-3a Approve Long Lead Procurements & FY 2015 \\
CD-2 Approve Performance Baseline & FY 2016 \\
CD-3 Approve Start of Construction & FY 2017 \\
CD-4 Approve Project Completion & FY 2020 \\
\hline \hline
\end{tabular}

\subsection{Cost}

Table 6.2: Fiscal year project funding profile assuming a TPC of $\$ 100 \mathrm{M}$.

\begin{tabular}{|l|c|c|c|c|c|c|c|}
\hline \hline & 2015 & 2016 & 2017 & 2018 & 2019 & 2020 & Total \\
\hline Total Project Cost (TPC) & 10.0 & 20 & 25 & 25 & 15 & 5 & 100.0 \\
\hline \hline
\end{tabular}

\subsection{Environment, Safety and Health}

The environmental impact of construction and operation of LUSI-II instrumentation is well within the range of past construction and operating activities at SLAC. Based on LCLS experience, no significant impact due to LUSI-II construction and subsequent operation is anticipated.

LUSI-II poses no qualitatively new hazards to personnel or the environment as compared to the hazards associated with current LCLS instrumentation. These hazards are well described in the LCLS Safety Assessment Documents. LUSI-II will comply with LCLS safety policies and protocols in addition to those formulated by SLAC ESH\&Q.

\subsection{Risk Management}

LUSI-II will follow the established SLAC risk management process and procedures applied to all projects at the lab. Through the conceptual design phase of the project, 
major technical, cost, schedule, safety, and management risks will be identified and carefully assessed and appropriate mitigation measures will be implemented. Rigorous risk management will be a continuous dynamic process through the life cycle of the project.

\subsubsection{Technical Risks}

The LUSI-II instruments will be based primarily on technology proven at LCLS-I. Thus, the technical risks associated with LUSI-II are minimal. Nonetheless, a few items carry an increased level of technical risk. In particular:

- Construction of low-noise, large-area detectors for the soft and hard x-ray regimes

- Mechanical stability of hard and soft x-ray split-and-delay devices

- Damage resistance of optical components when exposed to per-pulse energies greater than $10 \mathrm{~mJ}$

A risk mitigation plan will be developed to address research and development (R\&D) needed to mitigate all major technical risks. This plan will take into consideration the considerable R\&D currently being supported via LCLS-I operations.

\subsubsection{Cost Risks}

The LUSI-II overall cost risk is considered low at this time. Many of the LUSI-II instrument components (diagnostics, optics, lasers, detectors) will be based on minor modification or outright copies of LUSI-I designs, for which recent actual costs are thoroughly documented.

\subsubsection{Schedule Risks}

Based on LUSI best practices, installation of LUSI-II instruments will be phased to accommodate "early science" operation. This approach will be planned to avoid adverse impact on the overall LUSI-II installation activities. In addition, this installation will not impact, nor will it be impacted by, LCLS-I operation or LCLS-II electron/FEL commissioning activities. However, LCLS-II early science operation and remaining LUSI-II installation activities must be carefully coordinated because interferences can occur. As such, there are no major external factors that pose a significant schedule risk besides delays in project funding. 


\section{REFERENCES}

[1] S. Baradaran, U. Bergmann, H. Dürr, K. Gaffney, J. Goldstein, M. Gühr, J. Hastings, P. Heimann, R. Lee, M. Seibert, and J. Stöhr, editors. LCLS-II New Instruments Workshops Report. SLAC Report SLAC-R-993, 2012.

[2] J. Stöhr, editor. Science driven instrumentation for LCLS-II, 2012.

[3] See http://science.energy.gov/ /media/bes/besac/pdf/Ngps_rpt.pdf.

[4] L Young, EP Kanter, B Krässig, Y Li, AM March, ST Pratt, R Santra, SH Southworth, N Rohringer, LF DiMauro, et al. Femtosecond electronic response of atoms to ultra-intense x-rays. Nature, 466(7302):56-61, 2010.

[5] M. Hoener, L. Fang, O. Kornilov, O. Gessner, S. T. Pratt, M. Gühr, E. P. Kanter, C. Blaga, C. Bostedt, J. D. Bozek, P. H. Bucksbaum, C. Buth, M. Chen, R. Coffee, J. Cryan, L. DiMauro, M. Glownia, E. Hosler, E. Kukk, S. R. Leone, B. McFarland, M. Messerschmidt, B. Murphy, V. Petrovic, D. Rolles, and N. Berrah. Ultraintense x-ray induced ionization, dissociation, and frustrated absorption in molecular nitrogen. Phys. Rev. Lett., 104:253002, Jun 2010 .

[6] Stefan P. Hau-Riege, Richard M. Bionta, Dmitri D. Ryutov, Richard A. London, Elden Ables, Keith I. Kishiyama, Stewart Shen, Mark A. McKernan, Donn H. McMahon, Marc Messerschmidt, Jacek Krzywinski, Peter Stefan, James Turner, and Beata Ziaja. Near-ultraviolet luminescence of $N_{2}$ irradiated by short x-ray pulses. Phys. Rev. Lett., 105:043003, Jul 2010.

[7] James P. Cryan, J. M. Glownia, J. Andreasson, A. Belkacem, N. Berrah, C. I. Blaga, C. Bostedt, J. Bozek, C. Buth, L. F. DiMauro, L. Fang, O. Gessner, M. Guehr, J. Hajdu, M. P. Hertlein, M. Hoener, O. Kornilov, J. P. Marangos, A. M. March, B. K. McFarland, H. Merdji, V. S. Petrović, C. Raman, D. Ray, D. Reis, F. Tarantelli, M. Trigo, J. L. White, W. White, L. Young, P. H. Bucksbaum, and R. N. Coffee. Auger electron angular distribution of double core-hole states in the molecular reference frame. Phys. Rev. Lett., 105:083004, Aug 2010.

[8] L. Fang, M. Hoener, O. Gessner, F. Tarantelli, S. T. Pratt, O. Kornilov, C. Buth, M. Gühr, E. P. Kanter, C. Bostedt, J. D. Bozek, P. H. Bucksbaum, M. Chen, R. Coffee, J. Cryan, M. Glownia, E. Kukk, S. R. Leone, 
and N. Berrah. Double core-hole production in $N_{2}$ : Beating the Auger clock. Phys. Rev. Lett., 105:083005, Aug 2010.

[9] S. P. Hau-Riege, R. A. London, A. Graf, S. L. Baker, R. Soufli, R. Sobierajski, T. Burian, J. Chalupsky, L. Juha, J. Gaudin, J. Krzywinski, S. Moeller, M. Messerschmidt, J. Bozek, and C. Bostedt. Interaction of short x-ray pulses with low-z x-ray optics materials at the LCLS free-electron laser. Opt. Express, 18(23):23933-23938, Nov 2010.

[10] G. Doumy, C. Roedig, S.-K. Son, C. I. Blaga, A. D. DiChiara, R. Santra, N. Berrah, C. Bostedt, J. D. Bozek, P. H. Bucksbaum, J. P. Cryan, L. Fang, S. Ghimire, J. M. Glownia, M. Hoener, E. P. Kanter, B. Krässig, M. Kuebel, M. Messerschmidt, G. G. Paulus, D. A. Reis, N. Rohringer, L. Young, P. Agostini, and L. F. DiMauro. Nonlinear atomic response to intense ultrashort $\mathrm{x}$ rays. Phys. Rev. Lett., 106:083002, Feb 2011.

[11] Philip H Bucksbaum, Ryan Coffee, and Nora Berrah. The first atomic and molecular experiments at the Linac Coherent Light Source x-ray free electron laser. Advances in Atomic Molecular and Optical Physics, 60:239-289, 2011.

[12] Nora Berrah, Li Fang, Brendan Murphy, Timur Osipov, Kiyoshi Ueda, Edwin Kukk, Raimund Feifel, Peter van der Meulen, Peter Salen, Henning T. Schmidt, Richard D. Thomas, Mats Larsson, Robert Richter, Kevin C. Prince, John D. Bozek, Christoph Bostedt, Shin-ichi Wada, Maria N. Piancastelli, Motomichi Tashiro, and Masahiro Ehara. Double-core-hole spectroscopy for chemical analysis with an intense x-ray femtosecond laser. Proceedings of the National Academy of Sciences, 108(41):16912-16915, 2011.

[13] SM Vinko, O Ciricosta, BI Cho, K Engelhorn, H-K Chung, CRD Brown, T Burian, J Chalupskỳ, RW Falcone, C Graves, et al. Creation and diagnosis of a solid-density plasma with an x-ray free-electron laser. Nature, 482(7383):59$62,2012$.

[14] Benedikt Rudek, Sang-Kil Son, Lutz Foucar, Sascha W Epp, Benjamin Erk, Robert Hartmann, Marcus Adolph, Robert Andritschke, Andrew Aquila, Nora Berrah, et al. Ultra-efficient ionization of heavy atoms by intense x-ray freeelectron laser pulses. Nature Photonics, 2012.

[15] Sebastian Schorb, Daniela Rupp, Michelle L. Swiggers, Ryan N. Coffee, Marc Messerschmidt, Garth Williams, John D. Bozek, Shin-Ichi Wada, Oleg Kornilov, Thomas Möller, and Christoph Bostedt. Size-dependent ultrafast ionization dynamics of nanoscale samples in intense femtosecond x-ray freeelectron-laser pulses. Phys. Rev. Lett., 108:233401, Jun 2012.

[16] Nina Rohringer, Duncan Ryan, Richard A London, Michael Purvis, Felicie Albert, James Dunn, John D Bozek, Christoph Bostedt, Alexander Graf, Randal Hill, et al. Atomic inner-shell x-ray laser at 1.46 nanometres pumped by an x-ray free-electron laser. Nature, 481(7382):488-491, 2012. 
[17] M Marvin Seibert, Tomas Ekeberg, Filipe RNC Maia, Martin Svenda, Jakob Andreasson, Olof Jönsson, Duško Odić, Bianca Iwan, Andrea Rocker, Daniel Westphal, et al. Single mimivirus particles intercepted and imaged with an x-ray laser. Nature, 470(7332):78-81, 2011.

[18] ND Loh, CY Hampton, AV Martin, D Starodub, RG Sierra, A Barty, A Aquila, J Schulz, L Lomb, J Steinbrener, et al. Fractal morphology, imaging and mass spectrometry of single aerosol particles in flight. Nature, 486(7404):513-517, 2012 .

[19] M Dell'Angela, T Anniyev, M Beye, R Coffee, A Föhlisch, J Gladh, T Katayama, S Kaya, O Krupin, J LaRue, et al. Real-time observation of surface bond breaking with an x-ray laser. Science, 339(6125):1302-1305, 2013.

[20] Tianhan Wang, Diling Zhu, Benny Wu, Catherine Graves, Stefan Schaffert, Torbjörn Rander, Leonard Müller, Boris Vodungbo, Cédric Baumier, David P Bernstein, et al. Femtosecond single-shot imaging of nanoscale ferromagnetic order in $\mathrm{Co} / \mathrm{Pd}$ multilayers using resonant x-ray holography. Physical Review Letters, 108(26):267403, 2012.

[21] Wei-Sheng Lee, YD Chuang, RG Moore, Y Zhu, L Patthey, M Trigo, DH Lu, PS Kirchmann, O Krupin, M Yi, et al. Phase fluctuations and the absence of topological defects in a photo-excited charge-ordered nickelate. Nature Communications, 3:838, 2012.

[22] C. E. Graves, A. H. Reid, T. Wang, B. Wu, S. de Jong, K. Vahaplar, I. Radu, D. P. Bernstein, M. Messerschmidt, L. Muller, R. Coffee, M. Bionta, S. W. Epp, R. Hartmann, N. Kimmel, et al. Nanoscale spin reversal by non-local angular momentum transfer following ultrafast laser excitation in ferrimagnetic GdFeCo. Nature Materials, 12(4):293-298, 2013.

[23] TE Glover, DM Fritz, M Cammarata, TK Allison, Sinisa Coh, JM Feldkamp, H Lemke, D Zhu, Y Feng, RN Coffee, et al. X-ray and optical wave mixing. Nature, 488(7413):603-608, 2012.

[24] Dan Daranciang, Matthew J. Highland, Haidan Wen, Steve M. Young, Nathaniel C. Brandt, Harold Y. Hwang, Michael Vattilana, Matthieu Nicoul, Florian Quirin, John Goodfellow, Tingting Qi, Ilya Grinberg, David M. Fritz, Marco Cammarata, Diling Zhu, Henrik T. Lemke, Donald A. Walko, Eric M. Dufresne, Yuelin Li, Jörgen Larsson, David A. Reis, Klaus Sokolowski-Tinten, Keith A. Nelson, Andrew M. Rappe, Paul H. Fuoss, G. Brian Stephenson, and Aaron M. Lindenberg. Ultrafast photovoltaic response in ferroelectric nanolayers. Phys. Rev. Lett., 108:087601, Feb 2012.

[25] S. O. Hruszkewycz, M. Sutton, P. H. Fuoss, B. Adams, S. Rosenkranz, K. F. Ludwig, W. Roseker, D. Fritz, M. Cammarata, D. Zhu, S. Lee, H. Lemke, 
C. Gutt, A. Robert, G. Grübel, and G. B. Stephenson. High contrast xray speckle from atomic-scale order in liquids and glasses. Phys. Rev. Lett., 109:185502, Nov 2012.

[26] C. Gutt, P. Wochner, B. Fischer, H. Conrad, M. Castro-Colin, S. Lee, F. Lehmkühler, I. Steinke, M. Sprung, W. Roseker, D. Zhu, H. Lemke, S. Bogle, P. H. Fuoss, G. B. Stephenson, M. Cammarata, D. M. Fritz, A. Robert, and G. Grübel. Single shot spatial and temporal coherence properties of the SLAC Linac Coherent Light Source in the hard x-ray regime. Phys. Rev. Lett., 108:024801, Jan 2012.

[27] Mina R Bionta, HT Lemke, JP Cryan, JM Glownia, C Bostedt, M Cammarata, J-C Castagna, Y Ding, DM Fritz, AR Fry, et al. Spectral encoding of xray/optical relative delay. Optics Express, 19(22):21855-21865, 2011.

[28] O Krupin, M Trigo, WF Schlotter, M Beye, F Sorgenfrei, JJ Turner, DA Reis, N Gerken, S Lee, WS Lee, et al. Temporal cross-correlation of x-ray free electron and optical lasers using soft x-ray pulse induced transient reflectivity. Optics Express, 20(10):11396-11406, 2012.

[29] M Harmand, R Coffee, MR Bionta, M Chollet, D French, D Zhu, DM Fritz, HT Lemke, N Medvedev, B Ziaja, et al. Achieving few-femtosecond timesorting at hard x-ray free-electron lasers. Nature Photonics, 2013.

[30] Lars Redecke, Karol Nass, Daniel P DePonte, Thomas A White, Dirk Rehders, Anton Barty, Francesco Stellato, Mengning Liang, Thomas RM Barends, Sébastien Boutet, et al. Natively inhibited trypanosoma brucei cathepsin b structure determined by using an x-ray laser. Science, 339(6116):227-230, 2013.

[31] Henry N Chapman, Petra Fromme, Anton Barty, Thomas A White, Richard A Kirian, Andrew Aquila, Mark S Hunter, Joachim Schulz, Daniel P DePonte, Uwe Weierstall, et al. Femtosecond x-ray protein nanocrystallography. Nature, 470(7332):73-77, 2011.

[32] Sébastien Boutet, Lukas Lomb, Garth J Williams, Thomas RM Barends, Andrew Aquila, R Bruce Doak, Uwe Weierstall, Daniel P DePonte, Jan Steinbrener, Robert L Shoeman, et al. High-resolution protein structure determination by serial femtosecond crystallography. Science, 337(6092):362-364, 2012.

[33] Jan Kern, Roberto Alonso-Mori, Rosalie Tran, Johan Hattne, Richard J Gildea, Nathaniel Echols, Carina Glöckner, Julia Hellmich, Hartawan Laksmono, Raymond G Sierra, et al. Simultaneous femtosecond x-ray spectroscopy and diffraction of photosystem II at room temperature. Science, 2013.

[34] Chun Hong Yoon, Peter Schwander, Chantal Abergel, Inger Andersson, Jakob Andreasson, Andrew Aquila, Saša Bajt, Miriam Barthelmess, Anton Barty, 
Michael J Bogan, et al. Unsupervised classification of single-particle x-ray diffraction snapshots by spectral clustering. Optics Express, 19(17):16542$16549,2011$.

[35] Anton Barty, Carl Caleman, Andrew Aquila, Nicusor Timneanu, Lukas Lomb, Thomas A White, Jakob Andreasson, David Arnlund, Saša Bajt, Thomas RM Barends, et al. Self-terminating diffraction gates femtosecond x-ray nanocrystallography measurements. Nature Photonics, 6(1):35-40, 2011.

[36] Andrew Aquila, Mark S Hunter, R Bruce Doak, Richard A Kirian, Petra Fromme, Thomas A White, Jakob Andreasson, David Arnlund, Saša Bajt, Thomas RM Barends, et al. Time-resolved protein nanocrystallography using an x-ray free-electron laser. Optics Express, 20(3):2706-2716, 2012.

[37] Linda C Johansson, David Arnlund, Thomas A White, Gergely Katona, Daniel P DePonte, Uwe Weierstall, R Bruce Doak, Robert L Shoeman, Lukas Lomb, Erik Malmerberg, et al. Lipidic phase membrane protein serial femtosecond crystallography. Nature Methods, 9(3):263-265, 2012.

[38] Rudolf Koopmann, Karolina Cupelli, Lars Redecke, Karol Nass, Daniel P DePonte, Thomas A White, Francesco Stellato, Dirk Rehders, Mengning Liang, Jakob Andreasson, et al. In vivo protein crystallization opens new routes in structural biology. Nature Methods, 2012.

[39] Stephan Kassemeyer, Jan Steinbrener, Lukas Lomb, Elisabeth Hartmann, Andrew Aquila, Anton Barty, Andrew V Martin, Christina Y Hampton, Saša Bajt, Miriam Barthelmess, et al. Femtosecond free-electron laser x-ray diffraction data sets for algorithm development. Optics Express, 20(4):4149-4158, 2012 .

[40] Jan Kern, Roberto Alonso-Mori, Julia Hellmich, Rosalie Tran, Johan Hattne, Hartawan Laksmono, Carina Glöckner, Nathaniel Echols, Raymond G Sierra, Jonas Sellberg, et al. Room temperature femtosecond x-ray diffraction of photosystem II microcrystals. Proceedings of the National Academy of Sciences, 109(25):9721-9726, 2012.

[41] AV Martin, F Wang, ND Loh, T Ekeberg, FRNC Maia, M Hantke, G van der Schot, CY Hampton, RG Sierra, A Aquila, et al. Noise-robust coherent diffractive imaging with a single diffraction pattern. Optics Express, 20(15):1665016661, 2012.

[42] Raymond G Sierra, Hartawan Laksmono, Jan Kern, Rosalie Tran, Johan Hattne, Roberto Alonso-Mori, Benedikt Lassalle-Kaiser, Carina Glockner, Julia Hellmich, Donald W Schafer, et al. Nanoflow electrospinning serial femtosecond crystallography. Acta Crystallographica Section D: Biological Crystallography, 68(11):1584-1587, 2012. 
[43] D Starodub, A Aquila, S Bajt, M Barthelmess, A Barty, C Bostedt, JD Bozek, N Coppola, RB Doak, SW Epp, et al. Single-particle structure determination by correlations of snapshot x-ray diffraction patterns. Nature Communications, 3:1276, 2012.

[44] Elbio Dagotto. Complexity in strongly correlated electronic systems. Science, 309(5732):257-262, 2005.

[45] Steven A Kivelson, IP Bindloss, E Fradkin, V Oganesyan, JM Tranquada, Aharon Kapitulnik, and Craig Howald. How to detect fluctuating stripes in the high-temperature superconductors. Reviews of Modern Physics, 75(4):1201, 2003.

[46] Tom Timusk and Bryan Statt. The pseudogap in high-temperature superconductors: an experimental survey. Reports on Progress in Physics, 62(1):61, 1999.

[47] G Ghiringhelli, M Le Tacon, M Minola, S Blanco-Canosa, C Mazzoli, NB Brookes, GM De Luca, A Frano, DG Hawthorn, F He, et al. Longrange incommensurate charge fluctuations in (Y, Nd) $\mathrm{Ba}_{2} \mathrm{Cu}_{3} \mathrm{O}_{6+x}$. Science, 337(6096):821-825, 2012.

[48] J Chang, E Blackburn, AT Holmes, NB Christensen, J Larsen, J Mesot, Ruixing Liang, DA Bonn, WN Hardy, A Watenphul, et al. Direct observation of competition between superconductivity and charge density wave order in $\mathrm{YBa}_{2} \mathrm{Cu}_{3} \mathrm{O}_{7-d}$. Nature Physics, 8(12):871-876, 2012.

[49] Yoichi Kamihara, Hidenori Hiramatsu, Masahiro Hirano, Ryuto Kawamura, Hiroshi Yanagi, Toshio Kamiya, and Hideo Hosono. Iron-based layered superconductor: LaOFeP. Journal of the American Chemical Society, 128(31):10012-10013, 2006.

[50] Eduardo Fradkin and Steven A Kivelson. High-temperature superconductivity: Ineluctable complexity. Nature Physics, 8(12):864-866, 2012.

[51] Tao Wu, Hadrien Mayaffre, Steffen Krämer, Mladen Horvatić, Claude Berthier, WN Hardy, Ruixing Liang, DA Bonn, and Marc-Henri Julien. Magneticfield-induced charge-stripe order in the high-temperature superconductor $\mathrm{YBa}_{2} \mathrm{Cu}_{3} \mathrm{Oy}$. Nature, 477(7363):191-194, 2011.

[52] L. Braicovich, J. van den Brink, V. Bisogni, M. Moretti Sala, L. J. P. Ament, N. B. Brookes, G. M. De Luca, M. Salluzzo, T. Schmitt, V. N. Strocov, and G. Ghiringhelli. Magnetic excitations and phase separation in the underdoped $\mathrm{La}_{2-x} \mathrm{Sr}_{x} \mathrm{CuO} \mathrm{O}_{4}$ superconductor measured by resonant inelastic x-ray scattering. Phys. Rev. Lett., 104:077002, Feb 2010.

[53] Mathieu Le Tacon, G Ghiringhelli, Jiri Chaloupka, M Moretti Sala, V Hinkov, MW Haverkort, M Minola, M Bakr, KJ Zhou, S Blanco-Canosa, et al. Intense 
paramagnon excitations in a large family of high-temperature superconductors. Nature Physics, 7(9):725-730, 2011.

[54] V. Bisogni, M. Moretti Sala, A. Bendounan, N. B. Brookes, G. Ghiringhelli, and L. Braicovich. Bimagnon studies in cuprates with resonant inelastic X-ray scattering at the $\mathrm{O} K$ edge. II. Doping effect in $\mathrm{La}_{2-x} \mathrm{Sr}_{x} \mathrm{CuO}$. Phys. Rev. B, 85:214528, Jun 2012.

[55] S. Wakimoto, H. Kimura, K. Ishii, K. Ikeuchi, T. Adachi, M. Fujita, K. Kakurai, Y. Koike, J. Mizuki, Y. Noda, K. Yamada, A. H. Said, and Yu. Shvyd'ko. Charge excitations in the stripe-ordered $\mathrm{La}_{5 / 3} \mathrm{Sr}_{1 / 3} \mathrm{NiO}_{4}$ and $\mathrm{La}_{2-x}(\mathrm{Ba}, \mathrm{Sr})_{x} \mathrm{CuO} \mathrm{O}_{4}$ superconducting compounds. Phys. Rev. Lett., 102:157001, Apr 2009.

[56] Nicolas Doiron-Leyraud, Cyril Proust, David LeBoeuf, Julien Levallois, JeanBaptiste Bonnemaison, Ruixing Liang, DA Bonn, WN Hardy, and Louis Taillefer. Quantum oscillations and the Fermi surface in an underdoped high-Tc superconductor. Nature, 447(7144):565-568, 2007.

[57] Scott C Riggs, O Vafek, JB Kemper, JB Betts, A Migliori, FF Balakirev, WN Hardy, Ruixing Liang, DA Bonn, and GS Boebinger. Heat capacity through the magnetic-field-induced resistive transition in an underdoped hightemperature superconductor. Nature Physics, 7(4):332-335, 2011.

[58] D Fausti, RI Tobey, N Dean, S Kaiser, A Dienst, MC Hoffmann, S Pyon, T Takayama, H Takagi, and A Cavalleri. Light-induced superconductivity in a stripe-ordered cuprate. Science, 331(6014):189-191, 2011.

[59] Wei-Sheng Lee, YD Chuang, RG Moore, Y Zhu, L Patthey, M Trigo, DH Lu, PS Kirchmann, O Krupin, M Yi, et al. Phase fluctuations and the absence of topological defects in a photo-excited charge-ordered nickelate. Nature Communications, 3:838, 2012.

[60] S. L. Johnson, R. A. de Souza, U. Staub, P. Beaud, E. Möhr-Vorobeva, G. Ingold, A. Caviezel, V. Scagnoli, W. F. Schlotter, J. J. Turner, O. Krupin, W.-S. Lee, Y.-D. Chuang, L. Patthey, R. G. Moore, D. Lu, M. Yi, P. S. Kirchmann, M. Trigo, P. Denes, D. Doering, Z. Hussain, Z.-X. Shen, D. Prabhakaran, and A. T. Boothroyd. Femtosecond dynamics of the collinear-to-spiral antiferromagnetic phase transition in CuO. Phys. Rev. Lett., 108:037203, Jan 2012.

[61] M Först, RI Tobey, S Wall, H Bromberger, V Khanna, AL Cavalieri, Y-D Chuang, WS Lee, R Moore, WF Schlotter, et al. Driving magnetic order in a manganite by ultrafast lattice excitation. Physical Review B, 84(24):241104, 2011.

[62] WS Lee, S Johnston, B Moritz, J Lee, M Yi, KJ Zhou, T Schmitt, L Patthey, V Strocov, K Kudo, et al. The role of lattice coupling in establishing electronic 
and magnetic properties in quasi-one-dimensional cuprates. arXiv preprint arXiv:1301.4267, 2013.

[63] BN Brockhouse and AT Stewart. Scattering of neutrons by phonons in an aluminum single crystal. Physical Review, 100(2):756, 1955.

[64] Jean-Pascal Rueff and Abhay Shukla. Inelastic x-ray scattering by electronic excitations under high pressure. Rev. Mod. Phys., 82:847-896, Mar 2010.

[65] M. Krisch and F. Sette. Light scattering in solids IX, volume 108. Springer, 2007.

[66] P. Abbamonte, K. D. Finkelstein, M. D. Collins, and S. M. Gruner. Imaging density disturbances in water with a 41.3-attosecond time resolution. Phys. Rev. Lett., 92:237401, Jun 2004.

[67] Léon Van Hove. Correlations in space and time and born approximation scattering in systems of interacting particles. Phys. Rev., 95:249-262, Jul 1954.

[68] R. J. Glauber. Time-dependent displacement correlations and inelastic scattering by crystals. Phys. Rev., 98:1692-1698, Jun 1955.

[69] M Trigo, M Fuchs, J Chen, , et al. Direct measurement of time-dependent density-density correlations in a solid through the acoustic analog of the dynamical Casimir effect. arXiv preprint arXiv:1301.3503, 2013.

[70] Zheng Yang, Changhyun Ko, and Shriram Ramanathan. Oxide electronics utilizing ultrafast metal-insulator transitions. Annual Review of Materials Research, 41:337-367, 2011.

[71] G. Binasch, P. Grünberg, F. Saurenbach, and W. Zinn. Enhanced magnetoresistance in layered magnetic structures with antiferromagnetic interlayer exchange. Phys. Rev. B, 39:4828-4830, Mar 1989.

[72] M. N. Baibich, J. M. Broto, A. Fert, F. Nguyen Van Dau, F. Petroff, P. Etienne, G. Creuzet, A. Friederich, and J. Chazelas. Giant magnetoresistance of (001)Fe/(001)Cr magnetic superlattices. Phys. Rev. Lett., 61:2472-2475, Nov 1988.

[73] I Radu, K Vahaplar, C Stamm, T Kachel, N Pontius, HA Dürr, TA Ostler, J Barker, RFL Evans, RW Chantrell, et al. Transient ferromagnetic-like state mediating ultrafast reversal of antiferromagnetically coupled spins. Nature, 472(7342):205-208, 2011.

[74] Stefan Mathias, La-O Chan, Patrik Grychtol, Patrick Granitzka, Emrah Turgut, Justin M Shaw, Roman Adam, Hans T Nembach, Mark E Siemens, Steffen Eich, et al. Probing the timescale of the exchange interaction in a ferromagnetic alloy. Proceedings of the National Academy of Sciences, 109(13):4792-4797, 2012. 
[75] Michael Wulff, Anton Plech, Laurent Eybert, Rudolf Randler, Friedrich Schotte, and Philip Anfinrud. The realization of sub-nanosecond pump and probe experiments at the ESRF. Faraday Discuss., 122:13-26, 2003.

[76] Anthony R Cashmore, Jose A Jarillo, Ying-Jie Wu, and Dongmei Liu. Cryptochromes: blue light receptors for plants and animals. Science, 284(5415):760765, 1999.

[77] Nathan C Rockwell and J Clark Lagarias. A brief history of phytochromes. Chem. Phys. Chem., 11(6):1172-1180, 2010.

[78] Aziz Sancar et al. Structure and function of DNA photolyase and cryptochrome blue-light photoreceptors. Chemical Reviews-Columbus, 103(6):2203-2238, 2003.

[79] Li Song, MA El-Sayed, JK Lanyi, et al. Protein catalysis of the retinal subpicosecond photoisomerization in the primary process of bacteriorhodopsin photosynthesis. Science (New York, NY), 261(5123):891, 1993.

[80] Michael A van der Horst and Klaas J Hellingwerf. Photoreceptor proteins, "star actors of modern times": a review of the functional dynamics in the structure of representative members of six different photoreceptor families. Accounts of chemical research, 37(1):13-20, 2004.

[81] Annemarie B Wohri, Gergely Katona, Linda C Johansson, Emelie Fritz, Erik Malmerberg, Magnus Andersson, Jonathan Vincent, Mattias Eklund, Marco Cammarata, Michael Wulff, et al. Light-induced structural changes in a photosynthetic reaction center caught by Laue diffraction. Science Signalling, 328(5978):630, 2010.

[82] P Hamm, M Zurek, T Röschinger, H Patzelt, D Oesterhelt, and W Zinth. Femtosecond spectroscopy of the photoisomerisation of the protonated Schiff base of all-trans retinal. Chemical physics letters, 263(5):613-621, 1996.

[83] GR Fleming, JL Martin, and J Breton. Rates of primary electron transfer in photosynthetic reaction centres and their mechanistic implications. Nature, 333:190-192, 1988.

[84] Lele Duan, Fernando Bozoglian, Sukanta Mandal, Beverly Stewart, Timofei Privalov, Antoni Llobet, and Licheng Sun. A molecular ruthenium catalyst with water-oxidation activity comparable to that of photosystem II. Nature Chemistry, 4(5):418-423, 2012.

[85] Javier J Concepcion, Jonah W Jurss, M Kyle Brennaman, Paul G Hoertz, Antonio Otávio T Patrocinio, Neyde Yukie Murakami Iha, Joseph L Templeton, and Thomas J Meyer. Making oxygen with ruthenium complexes. Accounts of chemical research, 42(12):1954-1965, 2009. 
[86] Michael R Wasielewski. Energy, charge, and spin transport in molecules and self-assembled nanostructures inspired by photosynthesis. The Journal of organic chemistry, 71(14):5051-5066, 2006.

[87] Roberto Alonso-Mori, Jan Kern, Richard J. Gildea, Dimosthenis Sokaras, Tsu-Chien Weng, Benedikt Lassalle-Kaiser, Rosalie Tran, Johan Hattne, Hartawan Laksmono, Julia Hellmich, Carina Glöckner, Nathaniel Echols, Raymond G. Sierra, Donald W. Schafer, Jonas Sellberg, Christopher Kenney, Ryan Herbst, Jack Pines, Philip Hart, Sven Herrmann, Ralf W. Grosse-Kunstleve, Matthew J. Latimer, Alan R. Fry, Marc M. Messerschmidt, Alan Miahnahri, M. Marvin Seibert, Petrus H. Zwart, William E. White, Paul D. Adams, Michael J. Bogan, Sebastien Boutet, Garth J. Williams, Athina Zouni, Johannes Messinger, Pieter Glatzel, Nicholas K. Sauter, Vittal K. Yachandra, Junko Yano, and Uwe Bergmann. Energy-dispersive x-ray emission spectroscopy using an x-ray free-electron laser in a shot-by-shot mode. Proceedings of the National Academy of Sciences, 109(47):19103-19107, 2012.

[88] Yulia Pushkar, Xi Long, Pieter Glatzel, Gary W Brudvig, G Charles Dismukes, Terrence J Collins, Vittal K Yachandra, Junko Yano, and Uwe Bergmann. Direct detection of oxygen ligation to the $\mathrm{Mn}_{4} \mathrm{Ca}$ cluster of photosystem II by x-ray emission spectroscopy. Angewandte Chemie International Edition, 49(4):800-803, 2010.

[89] M Haumann, P Liebisch, C Müller, M Barra, M Grabolle, and H Dau. Photosynthetic $\mathrm{O}_{2}$ formation tracked by time-resolved x-ray experiments. Science, 310(5750):1019-1021, 2005.

[90] Yasufumi Umena, Keisuke Kawakami, Jian-Ren Shen, and Nobuo Kamiya. Crystal structure of oxygen-evolving photosystem II at a resolution of $1.9 \AA$. Nature, 473(7345):55-60, 2011.

[91] Hanneli R Hudock, Benjamin G Levine, Alexis L Thompson, Helmut Satzger, D Townsend, N Gador, S Ullrich, Albert Stolow, and Todd J Martínez. Ab initio molecular dynamics and time-resolved photoelectron spectroscopy of electronically excited uracil and thymine. The Journal of Physical Chemistry A, 111(34):8500-8508, 2007.

[92] Chris T Middleton, Kimberly de La Harpe, Charlene Su, Yu Kay Law, Carlos E Crespo-Hernández, and Bern Kohler. DNA excited-state dynamics: from single bases to the double helix. Physical Chemistry, 60, 2009.

[93] Clélia Canuel, Michel Mons, François Piuzzi, Benjamin Tardivel, Iliana Dimicoli, and Mohamed Elhanine. Excited states dynamics of DNA and RNA bases: Characterization of a stepwise deactivation pathway in the gas phase. The Journal of chemical physics, 122:074316, 2005. 
[94] Hyuk Kang, Kang Taek Lee, Boyong Jung, Yeon Jae Ko, Seong Keun Kim, et al. Intrinsic lifetimes of the excited state of DNA and RNA bases. Journal of the American Chemical Society, 124(44):12958-12959, 2002.

[95] Juan José Serrano-Pérez, Remedios González-Luque, Manuela Merchán, and Luis Serrano-Andrés. On the intrinsic population of the lowest triplet state of thymine. The Journal of Physical Chemistry B, 111(41):11880-11883, 2007.

[96] Serhiy Perun, Andrzej L Sobolewski, and Wolfgang Domcke. Conical intersections in thymine. The Journal of Physical Chemistry A, 110(49):13238-13244, 2006 .

[97] Manuela Merchán and Luis Serrano-Andrés. Ultrafast internal conversion of excited cytosine via the lowest $\pi \pi^{*}$ electronic singlet state. Journal of the American Chemical Society, 125(27):8108-8109, 2003.

[98] Daniel Healion, Jason D Biggs, and Shaul Mukamel. Manipulating oneand two-dimensional stimulated-x-ray resonant-Raman signals in molecules by pulse polarizations. Physical Review A, 86(3):033429, 2012.

[99] Siegfried Lünnemann, Alexander I Kuleff, and Lorenz S Cederbaum. Charge migration following ionization in systems with chromophore-donor and amineacceptor sites. The Journal of chemical physics, 129:104305, 2008.

[100] Ananyo Bhattacharya. Protein structures: Structures of desire. Nature, 449:24-27, 2009.

[101] Erin EH Tran, Mario J Borgnia, Oleg Kuybeda, David M Schauder, Alberto Bartesaghi, Gabriel A Frank, Guillermo Sapiro, Jacqueline LS Milne, and Sriram Subramaniam. Structural mechanism of trimeric HIV-1 envelope glycoprotein activation. PLoS Pathogens, 8(7):e1002797, 2012.

[102] David Eisenberg and Mathias Jucker. The amyloid state of proteins in human diseases. Cell, 148(6):1188-1203, 2012.

[103] Arthur Laganowsky, Cong Liu, Michael R Sawaya, Julian P Whitelegge, Jiyong Park, Minglei Zhao, Anna Pensalfini, Angela B Soriaga, Meytal Landau, Poh K Teng, et al. Atomic view of a toxic amyloid small oligomer. Science, 335(6073):1228-1231, 2012.

[104] A. A. Lutman, R. Coffee, Y. Ding, Z. Huang, J. Krzywinski, T. Maxwell, M. Messerschmidt, and H.-D. Nuhn. Experimental demonstration of femtosecond two-color x-ray free-electron lasers. Phys. Rev. Lett., 110:134801, Mar 2013. 